\title{
Green fronthaul allocation and power management in Cloud-RAN
}

\author{
Yuan Sun ${ }^{1}$, Shidang $\mathrm{Li}^{2}$ and Luxi Yang ${ }^{1 *}$
}

\begin{abstract}
In cloud radio access network (Cloud-RAN), most previous research aims to reduce the energy consumption of the total system and ignores specific requirements of energy-saving in mobile users. For the meantime, some efforts have been made to devise efficient signal quantization techniques for fronthaul capacity restriction to improve the performance of Cloud-RAN. To improve the user-centric energy efficiency (EE), we propose two kinds of optimization algorithms in orthogonal frequency division multiple access-based Cloud-RAN in this paper, whereby Gaussian quantization and uniform scalar quantization methods are utilized. For single-RRH scenario, alternative iterative optimization algorithms are proposed to maximize users' EE, by jointly allocating resources. Furthermore, in more general scenario for multiple RRHs, authors investigate a modified particle swarm optimization (M-PSO) algorithm to solve more complex problems, which remain non-linear and non-convex. Simulation results demonstrate the proposed schemes in this paper outperform conventional solutions and improve EE of users.
\end{abstract}

Keywords: Cloud-RAN, Fronthaul capacity restriction, Signal quantization, User-centric EE, Joint optimization, Modified PSO

\section{Introduction}

\subsection{Motivation}

With the rapid increment of mobile network, it is estimated that mobile data traffic will rise approximately 1000 times from 2010 to 2020 , and the cost is predicted to decrease 100 times by 2020 [1]. The rapid increase of data traffic has brought about a fast-growing high burden on the existing communication system, which include more strict requirements on spectrum efficiency (SE), energy efficiency (EE), capital expenditures, and operating expenditures. In order to achieve above targets, China Mobile raised Cloud-RAN in [2], which is considered as one promising candidate for next-generation communication technologies [3].

Cloud-RAN is a cloud-based, green, clean, and collaborative radio access network [4]. Figure 1 shows a simplified diagram of a Cloud-RAN structure. In this paper, each single mobile user is corresponding to one or more remote radio heads (RRHs). RRHs, as limited function transceivers, are attached to the central cloud via a

*Correspondence: Ixyang@seu.edu.cn

'School of Information Science and Engineering, Southeast University, 210096 Nanjing, China

Full list of author information is available at the end of the article limited-capacity fronthaul link. The baseband unit (BBU) pool, also called as "cloud," consists of multiple BBUs, for the better adaptation to non-uniform traffic. The BBU pool is attached to the core internet via a backhaul link, through gateway relays. Different from a traditional communication system, Cloud-RAN requires fewer BBUs and saves network operating costs.

Energy-saving problem has attracted lots of attention in Cloud-RAN research field. But according to the authors knowledge, most of the previous research aim to reduce the energy consumption of the total system and ignore specific requirements of energy saving of mobile users [5-7]. To reduce the interference between different channels [8] and decrease design complexity, we introduce orthogonal frequency division multiple access (OFDMA) to Cloud-RAN systems in this paper. Nevertheless, the fronthaul link capacity of Cloud-RAN is limited, which is different from a conventional cellular system [9]. To further improve system performance of Cloud-RAN, signal quantization techniques have been applied in related research [10].

Overall, our motivation is to design resource allocation scheme to improve the energy efficiency of users, under "signal quantization" mechanism in Cloud-RAN system 


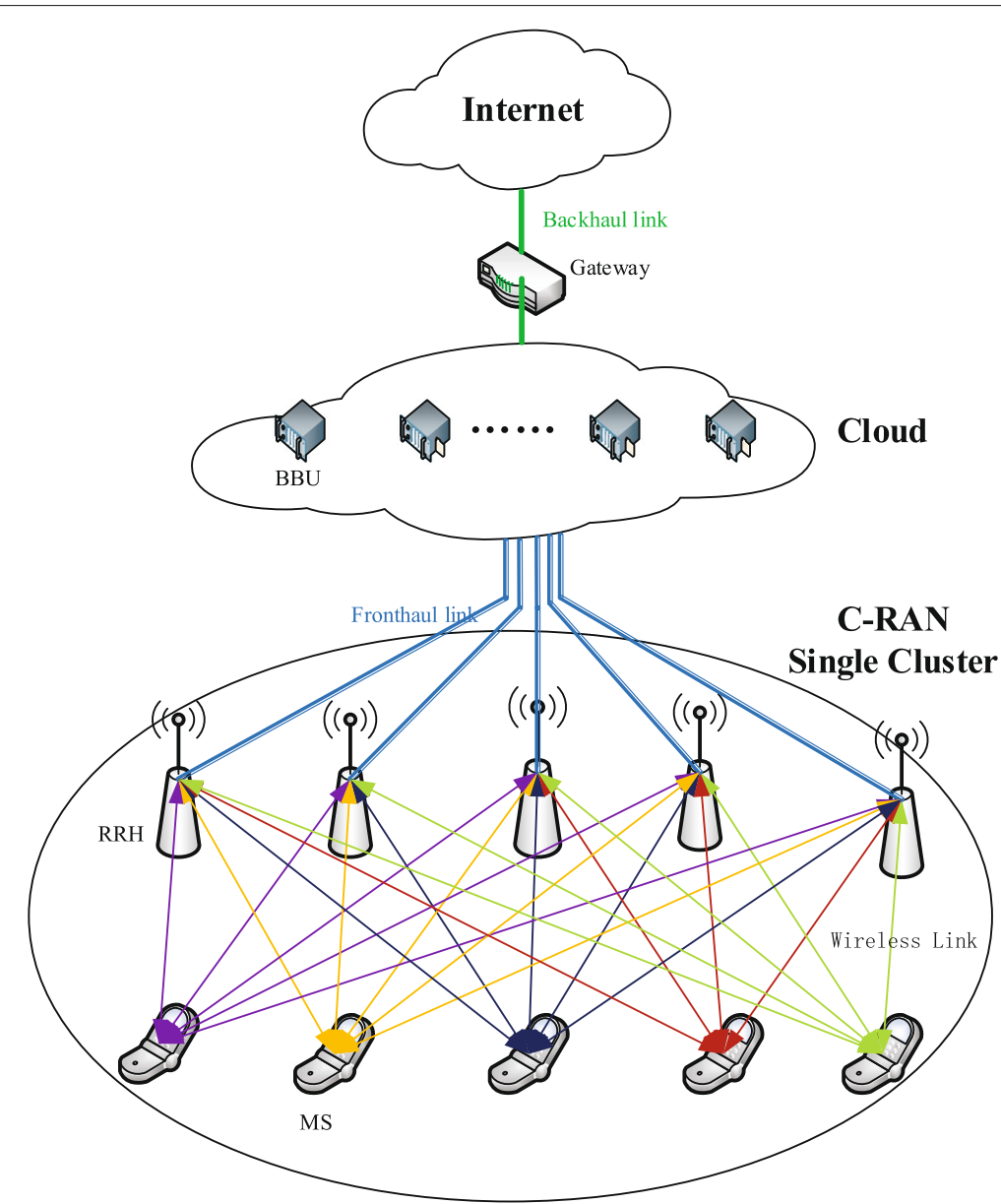

Fig. 1 An architecture layer diagram of Cloud-RAN

in this paper. In single-RRH scenario, authors use fractional programming and Lagrangian dual decomposition to jointly allocate power and fronthaul capacity. But in a more general scenario for multiple RRHs, the above optimization methods could not be directly utilized because of the more complex problems, in which objective functions and constraints also remain non-linear and non-convex. Instead, we need to seek a new optimization method to avoid slow convergence and high complexity. Actually, there exists a clear conflict between optimizing EE only and optimizing SE only. We will consider it in another paper, due to space limitation.

This paper focuses on the algorithm for jointly allocating user uplink transmit power and fronthaul capacity. The optimization process mainly occurs in the physical layer and does not know any design details of frames, including the number of signaling and specific bit values. Moreover, this article assumes the BBU pool knows all the information (including CSI); when all users do not move in the entire process, the users' association with RRHs does not change. In the process of resource allocation, there is little signaling overhead among cloud, RRHs, and users. Hence, we could not directly perform quantitative analysis of signaling overhead for Cloud-RAN systems. However, by using the related technologies, such as aggregation of $\mathrm{BBU}$ pool to increase the fault tolerance, "signal quantization/compression" for fronthaul link, the Cloud-RAN system could reduce the signaling overhead greatly.

\subsection{Related work}

In Cloud-RAN, the EE performance metric has attracted considerable attention because of the rapid growth of carbon emission and running cost of wireless communication systems [11, 12]. In [5], cross-layer resource management problem is formulated as a mixed-integer nonlinear programming problem, which could be relaxed into an easy-to-solve extended sum-utility maximization problem. Through jointly UL-DL (uplink-downlink) user association and beamforming design, a NP-hard problem of energy minimization is solved by converting to an original DL issue with two separate correlated subproblems for 
the original and virtual DL transmissions in Cloud-RAN [6]. Dai and Yu [13] compare the energy efficiencies of the data-sharing strategy and compression strategy by utilizing reweighted $l_{1}$ minimization and convex approximation. Wang et al. [14] introduce mobile cloud computing into Cloud-RAN, while authors establish a joint energysaving issue into a non-convex optimization with the constraints of task operating time, transmitting power, etc. In general, the above work pays attention to bring down the total energy consumption problems (unit: Joule), ignoring the specific energy-saving needs of the users (unit: $\mathrm{Bps} / \mathrm{W})$.

OFDMA, as a promising multi-access technique, has proved its advantages in both frequency and time domains to offer high throughput in the 4G system $[15,16]$. As mentioned above, we bring in OFDMA for the CloudRAN in this paper because of its advantages over interference avoidance and encoding design. Thus, complex beamforming design $[17,18]$ and interference cancellation are not needed in OFDMA-based Cloud-RAN in this paper. And it also has strong compatibility with the current 4G standards.

But in OFDMA-based Cloud-RAN system, on account of fronthaul capacity limitation, the conventional energysaving methods cannot be directly used. According to [2], the fronthaul traffic generated by a single user can easily reach multiple Gigabit byte per second (Gbps) because of the dense deployment of RRHs. And in practical CloudRAN system, a fiber link with magnitude of Gbps capacity is often utilized to transmit mobile data. Some signal compression/quantization methods have been studied in various networks $[8,10,19]$ to enhance the system performance and save fronthaul capacity resource. Furthermore, how to introduce effective "signal quanzation" techniques to user-centric energy-saving problem is still an open issue in OFDMA-based Cloud-RAN.

\subsection{Main contributions}

The contributions of this paper could be shown below:

- Different from the prior work, we aim to resolve the energy-saving issue of mobile users in an

OFDMA-based Cloud-RAN. In the UL transmission, under two different quantization methods, a joint resource allocation issue is formulated to maximize the energy efficiency of users, when two corresponding restrictions are user's UL transmission power and fronthaul capacity of RRHs. By solving the above problems, this paper intends to provide a new research direction for EE improvement in Cloud-RAN.

- In single-RRH scenario, this paper investigates a joint optimized resource allocation scheme to realize user-centric energy-saving goal, by utilizing Lagrange dual decomposition and iterative optimization method. Under Gaussian quantization and uniform scalar quantization, some useful insights on the optimal allocation plans can be obtained, respectively.

- In a more general scenario for multi-RRH, the similar optimization methods could not be directly utilized because of the more complex problems, in which objective functions and constraints also remain non-convex and non-linear. Instead, authors design a new Modified-PSO algorithm to solve this issue. Simulation results confirm the proposed schemes in this paper outperform conventional solutions.

\subsection{Organization}

The rest of this paper is organized as shown below. Section 2 discusses system model of Cloud-RAN and two quantization ways utilized in fronthaul signal processing. Section 3 solves the joint optimization problem for singleRRH scenario and provide some useful simulation results. Section 4 extends the single-RRH scenario to the general scenario for multi-RRH and deals with the joint optimization problem for multi-user. Finally, some concluding remarks are obtained in Section 5.

\section{System model and quantization schemes}

As shown in Fig. 1, the UL transmission in a single cluster of Cloud-RAN is considered. The core network, e.g., Internet, exists in the upper layer of Cloud-RAN. The central "cloud," consists of multiple BBUs, is connected to the core network via a backhaul link. The RRH is equipped with single antenna and its number is $M$, denoted as $\mathcal{M}=\{1, \ldots, M\}$. The number of single-antenna user is $K$, $\mathcal{K}=\{1, \ldots, K\}$. For each RRH $m, \forall m \in \mathcal{M}$, the capacity of fronthaul constraint is $T_{m}^{\text {total }}$ bps.

In the uplink transmission process, mobile users send signals to RRHs over wireless channel firstly. Second, each RRH quantizes its received signal and sends the corresponding digital codewords to the BBU pool. Third, the BBU pool joint decodes the users' messages carried on the signals from all the RRHs in the same cluster and upload the decoded messages through backhaul links.

The detailed models of signals and fronthaul link are showed as follows.

\subsection{Signal model at RRH in OFDMA-based Cloud-RAN}

In a system model, the total bandwidth $B \mathrm{~Hz}$, which is between $K$ users and $M$ RRHs, is divided equally over $N$ subcarriers (SCs), $\mathcal{N}=\{1, \ldots, N\}$. Single $\mathrm{SC} n$ is assigned to a single user simultaneously and $\Omega_{k}$ is denoted the SCs assigned to user $k, \forall k \in \mathcal{K}$.

The signals received by RRH $m$ at SC $n$ is defined as

$$
y_{m, n}=h_{m, k, n} \sqrt{p_{k, n}} s_{k, n}+z_{m, n},
$$

where

$n \in \Omega_{k}$ is the SC assigned to the user $k$, 
$h_{m, n, k}$ means the channel gain from $k$ th user to $m$ th $\mathrm{RRH}$ at $n$th $\mathrm{SC}$,

$p_{k, n}$ is the UL transmit power of $k$ th user,

$s_{k, n} \sim \mathcal{C N}(0,1)$ is the transmit signal of $k$ th user at $n$th $\mathrm{SC}$,

$z_{m, n} \sim \mathcal{C N}\left(0, \sigma_{m, n}^{2}\right)$ is the aggregation of additive white Gaussian noise (AWGN).

In this paper, we assume the SC allocation scheme $\Omega_{k}$ is scheduled for simplicity. In practical use, to improve the system performance, dynamic SC allocation scheme is often considered by allocating appropriate SCs to users of favorable wireless link conditions. We will pay more attention to more specific SC allocation strategies in future work.

Because the received symbol at SCs by each RRH is independent with each other, so we assume independent signal quantization at different RRHs. By utilizing a scalar quantization method [20], the quantized signal of $y_{m, n}$ could be rewritten as

$$
\tilde{y}_{m, n}=h_{m, k, n} \sqrt{p_{k, n}} s_{k, n}+z_{m, n}+e_{m, n}
$$

$e_{m, n}$ denotes the quantization error for the received symbol $y_{m, n}$ with zero mean and variance $q_{m, n}$.

After being quantified, $\tilde{y}_{m, n}$ is transformed into serial bits and sent to the BBU Pool via fronthaul link.

Utilizing maximal ratio combining (MRC) technique $[21,22]$, the SNR of $s_{k, n}$ is written as

$$
\gamma_{k, n}=\sum_{m=1}^{M}\left|h_{m, k, n}\right|^{2} p_{k, n} /\left(\sigma_{m, n}^{2}+q_{m, n}\right),
$$

where $n \in \Omega_{k}, k \in \mathcal{K}$.

\subsection{Two quantization schemes}

The first signal quantization method is Gaussian quantization (GQ). In Gaussian test channel (GTC) [23], $e_{m, n}$ given in (2) is Gaussian distributed, $e_{m, n} \sim \mathcal{C N}\left(0, q_{m, n}\right)$. According to [23], the transmission rate of $m$ th RRH's fronthaul link via $n$th SC to forward uplink transmission data is denoted as

$T_{m, n}^{(G)}=B \log _{2}\left(1+\left(\left|h_{m, k, n}\right|^{2} p_{k, n}+\sigma_{m, n}^{2}\right) / q_{m, n} / q_{m, n}\right) N N$

(G) superscript means variables in Gaussian test channel. Noise variance is included in the numerator of the second item in the bracket in the above formula (10). That is because the Gaussian white noise generated in the previous stage is used as part of the new signal during the generation of quantization errors at the RRH. Correspondingly, quantization noise becomes a noise component of the new signal to noise ratio.

The variance of $e_{m, n}$ of GQ could be rewritten as:

$$
q_{m, n}^{(G)}=\left(\left|h_{m, k, n}\right|^{2} p_{k, n}+\sigma_{m, n}^{2}\right) /\left(2^{N T_{m, n}^{(G)} / B}-1\right)
$$

The corresponding SNR obtained in (3) can be expressed as

$$
\gamma_{m, n}^{(G)}=\sum_{m=1}^{M} \frac{\left|h_{m, k, n}\right|^{2} p_{k, n}}{\sigma_{m, n}^{2}+\frac{\left|h_{m, k, n}\right|^{2} p_{k, n}+\sigma_{m, n}^{2}}{2^{N T_{m, n}^{(G)} / B}-1}}
$$

Based on rate-distortion theory, the above Gaussian quantization method has the best theoretical performance while it is hard to implement practically. In [8], a more practical uniform scalar quantization (USQ) scheme has been proposed. Due to space limitations, we just list the following conclusion referring the above GQ part. The transmission rate in RRH m's fronthaul link to forward the received data at $\mathrm{SC} n$ must satisfy the following constraint

$$
T_{m, n}^{(U)}=2 B D_{m, n}^{(U)} / N, D_{m, n} \in\{1,2, \ldots\}, \forall m \in M, n \in N
$$

$(U)$ superscript means variables in USQ scheme. The variance of the quantization error $e_{m, n}$ with USQ scheme can be expressed as

$$
q_{m, n}^{(U)}=3\left(\left|h_{m, k, n}\right|^{2} p_{k, n}+\sigma_{m, n}^{2}\right) 2^{-N T_{m, n}^{(U)} / B}
$$

The corresponding signal to noise ratio (SNR) with USQ scheme can be expressed as

$$
\gamma_{m, n}^{(U)}=\sum_{m=1}^{M} \frac{\left|h_{m, k, n}\right|^{2} p_{k, n}}{\sigma_{m, n}^{2}+3\left(\left|h_{m, k, n}\right|^{2} p_{k, n}+\sigma_{m, n}^{2}\right) 2^{-N T_{m, n}^{(U)} / B}}
$$

From the above expression, it can be seen the SNR of decoding $s_{k, n}$ in Cloud-RAN is also related to both users' allocation schemes $\left\{p_{k, n}\right\}$ and RRHs' fronthaul rate allocations $T_{k, n}^{(G)}, T_{k, n}^{(U)}$.

\section{Joint optimization of the single-RRH scenario}

In this subsection, $K$ is defined as 1 and $M$ is fixed to 1 , which cause the omission of $k$ and $m$. Authors define system bandwidth as $B$, user's UL power maximum value as $P^{\text {total }}$, RRH's fronthaul capacity restriction as $T^{\text {total }}$, and power loss of user except UL transmission power $p_{n}$ as $P_{c}$. The optimization models based on two quantization schemes are as follows 
$(P 1): \max _{\left\{p_{n}, T_{n}^{(G)}\right\}} \frac{B}{N} \frac{\sum_{n=1}^{N} \log _{2}\left(1+\frac{\left|h_{n}\right|^{2} p_{n}}{\sigma_{n}^{2}+\frac{\left|h_{n}\right|^{2}{ }^{2}+\sigma_{n}^{2}}{2^{N T_{n}(G)} / B_{-1}}}\right)}{\sum_{n=1}^{N} p_{n}+P_{c}}$

s.t.

$$
\sum_{n=1}^{N} p_{n} \leq P^{\text {total }}, \sum_{n=1}^{N} T_{n}^{(G)} \leq T^{\text {total }}, \forall n \in \mathcal{N}
$$

$$
(P 2): \max _{\left\{p_{n}, T_{n}^{(U)}\right\}} \frac{B}{N} \frac{\sum_{n=1}^{N} \log _{2}\left(1+\frac{\left|h_{n}\right|^{2} p_{n}}{\sigma_{n}^{2}+\frac{3 *\left(\left|h_{n}\right|^{2} p_{n}+\sigma_{n}^{2}\right)}{{ }_{2} T_{n}^{(U)} / B}}\right)}{\sum_{n=1}^{N} p_{n}+P_{c}}
$$

s.t.

$$
\begin{aligned}
& \sum_{n=1}^{N} p_{n} \leq P^{\text {total }}, \sum_{n=1}^{N} T_{n}^{(U)} \leq T^{\text {total }}, \forall n \in \mathcal{N} \\
& T_{n}^{(U)}=2 B D_{n}^{(U)} / N, D_{n} \in\{1,2, \ldots\}, \forall n \in \mathcal{N}
\end{aligned}
$$

Because (P1) and (P2) are both non-linear non-convex fractional programming problems, it's hard to obtain the global optimal solution directly. The following parts aim to solve two optimization problems separately and obtain some meaningful conclusions.

\subsection{Gaussian quantization}

Taking into account non-convex and non-linear characteristics, an alternating optimization method is adopted to convert original fractional programming issue to two relatively simple ones.

In (P1), the fronthaul capacity is assumed to have been allocated optimally and denoted $T_{n}^{(G)}=T_{n, o p t}^{(G)}$. Then origin (P1) is rewritten as

$$
\begin{aligned}
& \max _{\left\{p_{n}\right\}} \frac{B}{N} \frac{\sum_{n=1}^{N} \log _{2}\left(1+\frac{\left|h_{n}\right|^{2} p_{n}}{\sigma_{n}^{2}+\frac{\left|h_{n}\right|^{2} p_{n}+\sigma_{n}^{2}}{2^{N T_{n, o p t}^{(G)}}-1}}\right)}{\sum_{n=1}^{N} p_{n}+P_{c}} \\
& \text { s.t. } \sum_{n=1}^{N} p_{n} \leq P^{\text {total }}
\end{aligned}
$$

Define $\left\{p_{n}^{\text {opt }}\right\}$ as the optimal solution of new problem (15).

Correspondingly, UL transmit power is assumed to have been optimally allocated and denoted $p_{n}=p_{n}^{\text {opt }}$. Then origin (P1) is rewritten as

$$
\max _{\left\{T_{n}^{(G)}\right\}} \frac{B}{N} \frac{\sum_{n=1}^{N} \log _{2}\left(1+\frac{\left|h_{n}\right|^{2} p_{n}^{o p t}}{\sigma_{n}^{2}+\frac{\left|h_{n}\right|^{2} p_{n}^{o p t}+\sigma_{n}^{2}}{{ }_{2}^{N T_{n}^{(G)} / B}-1}}\right)}{\sum_{n=1}^{N} p_{n}^{o p t}+P_{c}}
$$

$$
\text { s.t. } \sum_{n=1}^{N} T_{n}^{(G)} \leq T^{\text {total }}
$$

Define $\left\{T_{n, o p t}^{(G)}\right\}$ as the optimal solution of new problem (17).

Next, before proposing iterative algorithms, authors will give the optimal solution of (15) and (17), respectively. Denote $\zeta=R\left(p_{n}, T_{n}^{(G)}\right) / P\left(p_{n}\right)$ as the EE performance of mobile users, $R\left(p_{n}, T_{n}^{(G)}\right)$ as the total throughput of users, $P\left(p_{n}\right)$ as the total power loss. Then, problem (P1) can be converted to $\max _{\left\{p_{n}, T_{n}^{(G)}\right\}} \zeta=R\left(p_{n}, T_{n}^{(G)}\right) / P\left(p_{n}\right)$.

In problem (17), the object function can be expressed as $\max \zeta=R\left(p_{n}\right) / P\left(p_{n}\right)$, which is a typical fractional $\left\{p_{n}\right\}$

programming problem. Using Lagrangian duality method in [24], we could offer the propositions as follows.

Proposition 3.1 The optimal conclusion of UL transmit power control in (15) could be written as:

$$
p_{n}^{o p t}=\left\{\begin{array}{ccc}
\frac{-\alpha_{n}+\sqrt{\alpha_{n}^{2}-4 \eta_{n}}}{2} & , \text { if }\left|h_{n}\right|^{2} / \sigma_{n}^{2}>f_{n}\left(T_{n, o p t}^{(G)}\right) \\
0 & , & \text { otherwise }
\end{array}\right.
$$

where

$$
\begin{aligned}
& \alpha_{n}=\frac{\left(2^{N T_{n, o p t}^{(G)} / B}+1\right) \sigma_{n}^{2}}{\left|h_{n}\right|^{2}}, \forall n \\
& \eta_{n}=\frac{2^{N T_{n, o p t}^{(G)} / B} \sigma_{n}^{4}}{\left|h_{n}\right|^{4}}-\frac{B \sigma_{n}^{2}\left(2^{N T_{n, o p t}^{(G)} / B}-1\right)}{\ln 2 N\left(\lambda^{(i)}+\zeta^{(i)}\right)\left|h_{n}\right|^{2}}, \forall i, n \\
& f_{n}\left(T_{n, o p t}^{(G)}\right)=\frac{\ln 2 N}{B} \frac{2^{N T_{n, o p t}^{(G)} / B}\left(\lambda^{(i)}+\zeta^{(i)}\right)}{2^{N T_{n, o p t}^{(G)} / B}-1}, \forall i, n \\
& \lambda^{(i+1)}=\left[\lambda^{(i)}-\chi_{\lambda}^{(i+1)} \times \nabla \lambda^{(i+1)}\right]^{+}, \forall i, n \\
& \nabla \lambda^{(i+1)}=P^{t o t a l}-\sum_{n=1}^{N} p_{n}^{(i)}, \forall i, n
\end{aligned}
$$

In (23), $\lambda^{(i)}$ is the duality factor for power constraint. $\chi_{\lambda}^{(i+1)}$ means the size of non-negative step. $\nabla \lambda^{(i+1)}$ represents the size of subgradient for $(i+1)$ th iteration.

Proof Please refer to Appendix A. 
Different from problem (15), problem (17) is convex when $T_{n, o p t}^{(G)}$ could be obtained as follows

Proposition 3.2 The optimal conclusion of fronthaul capacity in (17) could be written as:

$T_{n, \text { opt }}^{(G)}=\left\{\begin{array}{cl}\frac{B}{N} \log v_{n}+\frac{B}{N} \log _{2}\left(\frac{1-\beta p_{p}^{o p t}-\beta P_{c}}{\beta p_{n}^{\text {pt }}+\beta P_{c}}\right), & \text { if } v_{n}>\Theta_{n} \\ 0 & \text { otherwise }\end{array}\right.$

where

$$
\Theta_{n}=\frac{\beta p_{n}^{o p t}+\beta P_{c}}{1-\beta p_{n}^{o p t}-\beta P_{c}}, v_{n}=\frac{\left|h_{n}\right|^{2} p_{n}^{o p t}}{\sigma_{n}^{2}}, \beta<\frac{1}{p_{n}^{\text {opt }}+P_{c}}
$$

In the above expressions, $\beta$ is a constant which satisfies $\sum_{n=1}^{N} T_{n, o p t}^{(G)}=T^{\text {total }}$

Proof Consult Appendix B for details.

In Proposition 3.2, if the received SNR $v_{n}$ is below the threshold $\frac{\beta p_{n}^{o p t}+\beta P_{c}}{1-\beta p_{n}^{\text {ot }}-\beta P_{c}}, T_{n, o p t}^{(G)}$ is zero. That means the RRH need not transmit quantized signal to the BBU pool. Otherwise, if the received SNR $v_{n}$ is over the threshold, more quantized data need to be transmitted. The demand for fronthaul capacity is growing with higher values of $v_{n}$.

To solve problem (P1), we design the following algorithm based on Propositions 3.1 and 3.2:

Algorithm 1 Energy-efficient Fronthaul Rate Allocation And Power Allocation Under Gaussian Quantization for Problem (P1)

1: Set the maximum number of iterations $I_{\max }(1 \leq i \leq$ $\left.I_{\max }\right)$, starting value $\zeta^{(1)}$, converging factor $\varepsilon_{\zeta}$, and $p_{n}^{(1)}=P^{\text {total }} / N$,etc.

2: Set the iteration index initial value $i=1$.

3: $\quad$ For $1 \leq i \leq I_{\max }$

4: $\quad$ For $1 \leq n \leq N$

5: $\quad$ Calculate $T_{n, o p t}^{(G, i)}$ based on Proposition 3.2 with $p_{n}^{\text {opt }}=p_{n}^{(i)}$

6: $\quad$ Calculate $p_{n}^{(i, o p t)}$ based on Proposition 3.1 with $T_{n, o p t}^{(G)}=T_{n, o p t}^{(G, i)}, p_{n}^{(i)}=p_{n}^{(i, o p t)}$;

7: $\quad$ End For

8: $\quad$ Acquire $\zeta^{(i+1)}$ based on (10) in Problem (P1)

while $K=1, M=1$;

9: $\quad$ If $\zeta^{(i+1)}-\zeta^{(i)}<\varepsilon_{\zeta}$

10: $\quad$ break;

11: $\quad$ Else

12: $\quad i=i+1$; Go to step 4;

End If

14: $\quad$ End For
It must be particularly pointed out that the algorithm used in this paper is a centralized processing method, which is determined by the characteristics of CloudRAN itself. Compared with traditional communication systems, Cloud-RANs attenuate the performance of RRHs to reduce network deployment costs, with a formed BBU pool for more efficient centralized processing. Due to the following two reasons: (1) RRHs in Cloud-RAN only have limited functions, which could not meet the requirements of distributed processing; (2) Distributed processing requires a large amount of information to be exchanged among RRHs during the iterations of the algorithm. This will cause a lot of signaling overhead. We do not consider the usage of "distributed algorithms" in this paper.

\subsection{Uniform scalar quantization}

In the following sections, we aim to settle the problem (P2). Since the objective function of (P2) is similar to that in problem (P1), new integer constraint can be ignored firstly, then the propositions for problem (P1) could be redesigned for (P2). Therefore, an alternating optimization method is adopted to convert original fractional programming issue to two relatively simple ones. After solving two simple problems, the corresponding propositions are as follows.

Proposition 3.3 The optimal conclusion of UL transmit power control for problem (P2) which only contains power constraint could be written as:

$$
p_{n}^{o p t}=\left\{\begin{array}{ccc}
\frac{-\chi_{n}+\sqrt{\chi_{n}^{2}-4 \psi_{n}}}{2}, & \text { if } & \left|h_{n}\right|^{2} / \sigma_{n}^{2}>f_{n}\left(T_{n, o p t}^{(G)}\right) \\
0 & , & \text { otherwise }
\end{array}\right.
$$

where

$$
\begin{gathered}
\chi_{n}=\sigma_{n}^{2}\left(6+2^{N T_{n, o p t}^{(U)} / B}\right) / 3\left|h_{n}\right|^{2}, \forall n \\
\psi_{n}=\frac{\left(3+2^{N T_{n, o p t}^{(U)} / B}\right) \sigma_{n}^{4}}{3\left|h_{n}\right|^{4}}-\frac{B \sigma_{n}^{2} 2^{N T_{n, o p t}^{(U)} / B}}{3 \ln 2\left(\zeta^{(i)}+\lambda^{(i)}\right) N\left|h_{n}\right|^{2}}, \forall n, i \\
f_{n}\left(T_{n, o p t}^{(U)}\right)=\frac{N \ln 2}{B} \frac{\left(3+2^{N T_{n, o p t}^{(U)} / B}\right)\left(\zeta^{(i)}+\lambda^{(i)}\right)}{2^{N T_{n, o p t}^{(U)} / B}}, \forall n, i \\
\lambda^{(i+1)}=\left[\lambda^{(i)}-\chi_{\lambda}^{(i+1)} \times \nabla \lambda^{(i+1)}\right]^{+} \\
\nabla \lambda^{(i+1)}=P^{\text {total }}-\sum_{n=1}^{N} p_{n}^{(i)}, \forall i, n
\end{gathered}
$$


In (32), $\lambda^{(i)}$ is the duality factor for power constraint. $\chi_{\lambda}^{(i+1)}$ means the size of positive step. $\nabla \lambda^{(i+1)}$ represents the subgradient in the $(i+1)$ th iteration.

Proof Similar to Appendix A. Proof of proposition 3.1.

In Proposition 3.3, characteristic of optimal power allocation scheme $p_{n}^{o p t}$ with the given $T_{n, o p t}^{(U)}$ is similar to that in Proposition 3.1.

Proposition 3.4 The optimal conclusion of fronthaul rate allocation for problem (P2) which only contains fronthaul capacity constraint could be written as:

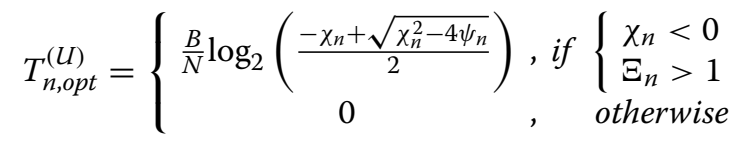

where

$$
\begin{aligned}
& \Xi_{n}=\frac{-\chi_{n}+\sqrt{\chi_{n}^{2}-4 \psi_{n}}}{2}, \forall n \\
& \chi_{n}=\frac{3\left|h_{n}\right|^{2} p_{n}^{o p t} \beta+6 \sigma_{n}^{2} \beta-3\left|h_{n}\right|^{2} p_{n}^{o p t}}{\sigma_{n}^{2} \beta}, \forall n \\
& \psi_{n}=\frac{9\left(\left|h_{n}\right|^{2} p_{n}^{o p t}+\sigma_{n}^{2}\right)}{\sigma_{n}^{2}}, \forall n
\end{aligned}
$$

and $\beta$ is a constant which satisfies $\sum_{n=1}^{N} T_{n, o p t}^{(U)}=T^{\text {total }}$.

Proof Similar to Appendix 6.2.

After obtaining Propositions 3.3 and 3.4, the new integer constraint $T_{m, n}^{(U)}=2 B D_{m, n}^{(U)} / N, D_{m, n} \in\{1,2, \ldots\}$ is considered in problem (P2). Based on Proposition 3.4, we input $p_{n}=p_{n}^{o p t}$ and output a feasible solution of $T_{n, o p t}^{(U)}$. Hence, we introduce a new variable $(0 \leq \tau \leq 1)$ to transform integer constraint as followed

$$
\begin{aligned}
& T_{n, \text { opt }}^{(U)}=\left\{\begin{array}{l}
\frac{2 B}{N} \Re_{n}, \text { if } \frac{N T_{n, o p t}^{(U)}-\Re_{n}<\tau}{2 B} \\
\frac{2 B}{N} \Im_{n}, \quad \text { otherwise }
\end{array}\right. \\
& \Re_{n}=\left\lfloor\frac{N T_{n, o p t}^{(U)}}{2 B}\right\rfloor, \Im_{n}=\left\lceil\frac{N T_{n, o p t}^{(U)}}{2 B}\right\rceil, \forall n
\end{aligned}
$$

$\Re_{n}$ is the number which rounds down to $N T_{n, o p t}^{(U)} / 2 B$, $\left\lceil N T_{n, o p t}^{(U)} / 2 B\right\rceil$ denotes the number which rounds up to $N T_{n, o p t}^{(U)} / 2 B$. For a given $\tau$, the original $T_{n, o p t}^{(U)}$ changes into $\frac{2 B}{N} \Re_{n}$ or $\frac{2 B}{N} \Im_{n}$. It could be observed the objective function in (P2) is monotonically increasing over $T_{n, o p t}^{(U)}$. Hence, we should find the optimal $\tau_{n, o p t}$ for each $T_{n, o p t}^{(U)}$ to ensure
$\sum_{n=1}^{N} T_{n, o p t}^{(U)}$ as large as possible within fronthaul constraint. For a group of input $\left\{T_{n, o p t}^{(U)}\right\}$, we need to design a corresponding group of output $\left\{\tau_{n, o p t}\right\}$, which contains $N$ different element. For simplicity, we set the same $\tau_{n, o p t}$ for all SCs and the subscript $n$ can be ignored. Bisection method could be used to find the optimal $\tau_{\text {opt }}$. Due to space limitations, we do not list specific algorithms for finding the optimal $\tau$ here. When $\tau=0$, there are still significant differences between the two quantization schemes, mainly because of different target formulas.

Based on Proposition 3.3, Proposition 3.4, a complete algorithm is redesigned to resolve (P2):

Algorithm 2 Energy-efficient Fronthaul Rate Allocation And Power Allocation Under Uniform Scalar Quantization for Problem (P2)

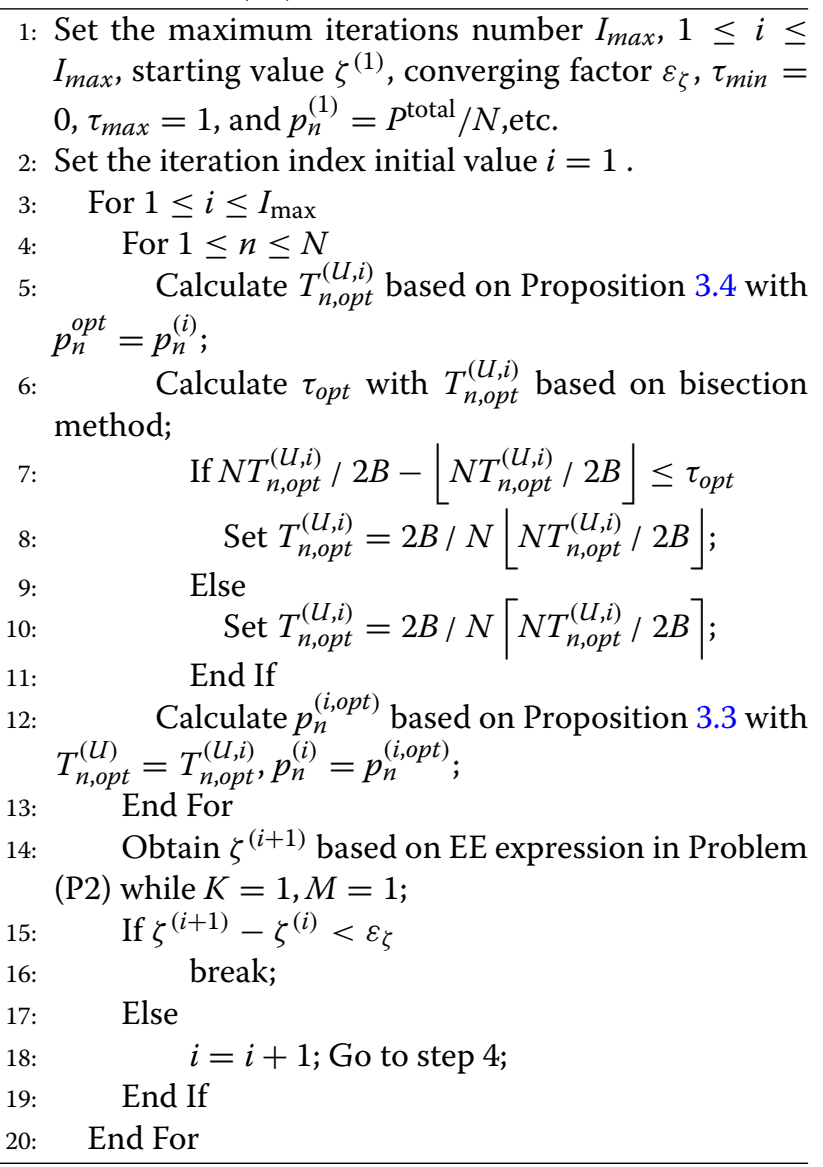

\subsection{Single-RRH scenario: numerical experiment}

To evaluate our proposed algorithms for single-RRH scenario $K=M=1$ and compare with other resource allocation methods, we present related simulation conclusions in this subpart. The rest of this subsection is organized as follows. Different schemes of resource allocation 
over SCs for two quantization schemes are analyzed in subsection C1. In subsection C2, we analyze the energy efficiency gain causing by different optimization methods for Gaussian test channel. Then, the effect of different parameter $\tau$ on energy efficiency gain under USQ method is listed in subsection C3.

\subsubsection{Power management and fronthaul capacity allocation over four SCs}

In this simulation part, bandwidth $B$ is set to $100 \mathrm{Mhz}$ and $N=4$. The channel gains are fixed as $\left|h_{1}\right|^{2}=1.28 \times 10^{-9}$, $\left|h_{2}\right|^{2}=6.12 \times 10^{-10},\left|h_{3}\right|^{2}=2.9 \times 10^{-11}$, and $\left|h_{4}\right|^{2}=$ $1.5 \times 10^{-11}$. The power spectral density of noise is $-169 \mathrm{dBm} / \mathrm{Hz}$. User UL maximum power limitation $P^{\text {total }}$ and maximum fronthaul limitation $T^{\text {total }}$ are set to $0.2 \mathrm{~W}$, $0.4 \mathrm{Gbps}$. In single-user subsection, power consumption of one user expect UL power is $P_{c} / N=0.5 \mathrm{~W} / 8=0.0625 \mathrm{~W}$. In Figs. 2 and 3, six resource allocation methods are compared as follows:

Method 1: GQ Max EE: Joint Optimization in this paper. In this scheme, we joint optimize its uplink transmit power and fronthaul rate to maximize user-centric energy efficiency with Gaussian quantization, as mentioned in Algorithm 1.

Method 2: GQ Max EE: Power Optimization only. In this scheme, the RRH allocate its fronthaul link capacity over all SCs equally. Then, under $T_{n}^{(G)}=T^{\text {total }} / N$, the user only optimize its uplink transmit power over SCs to maximize its energy efficiency.

Method 3: GQ Max EE: Fronthaul Optimization only. In this scheme, the user allocate its uplink transmit power over all SCs equally. Then, with $p_{n}=P^{\text {total }} / N$, the $\mathrm{RRH}$ only optimize its fronthaul link capacity over SCs to maximize user's energy efficiency.

Method 4: GQ Max Throughput: Joint Optimization. In this scheme, we reappear the optimization method in [8] and joint optimize its uplink transmit power and fronthaul capacity to maximize user's throughput instead of user's energy efficiency.

Method 5: Equal Fronthaul and Power Allocation. During this scheme, user allocates its uplink transmission power over all SCs equally when RRH allocates its fronthaul link capacity over all SCs equally.

Method 6: USQ Max EE: Joint Optimization in this paper. In this scheme, we joint optimize its uplink transmit power and fronthaul rate to maximize user-centric energy efficiency with uniform scalar quantization, as mentioned in Algorithm 2.

In Figs. 2 and 3, some useful conclusions could be obtained as follows.

Conclusion 1: In Fig. 2, method 1 and method 6 did not tend to allocate all power to SCs compared with methods 3-5. The power resource is almost allocated to two best channel quality subcarriers SC1 and SC2 in method 4. But for method 1 and method 6 , the total sum of the allocated power over four SCs is less than $P^{\text {total }}=0.2 \mathrm{~W}$. This states our designed Algorithm 1 and Algorithm 2 works well and saves the uplink transmit power effectively.

Conclusion 2: From Fig. 2, Fig. 3, we can observe that the joint optimization in method 1 and method 6 tend to allocate more power resource and fronthaul capacity to the better channel condition SC, which strategy is consistent

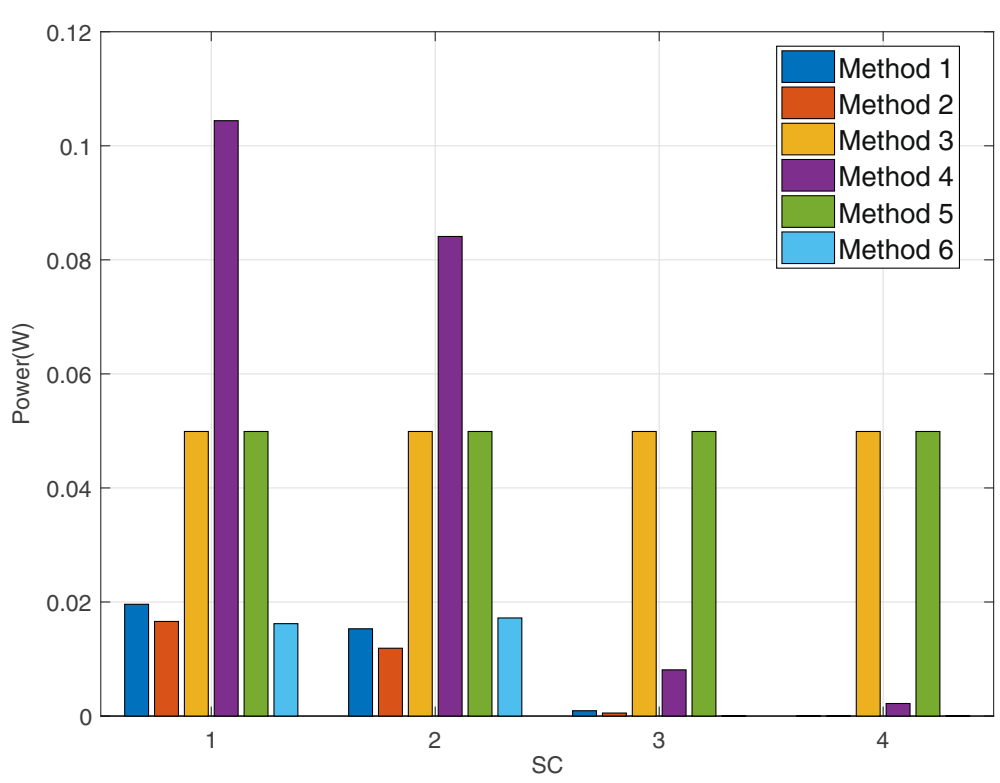

Fig. 2 Power allocation over four SCS 


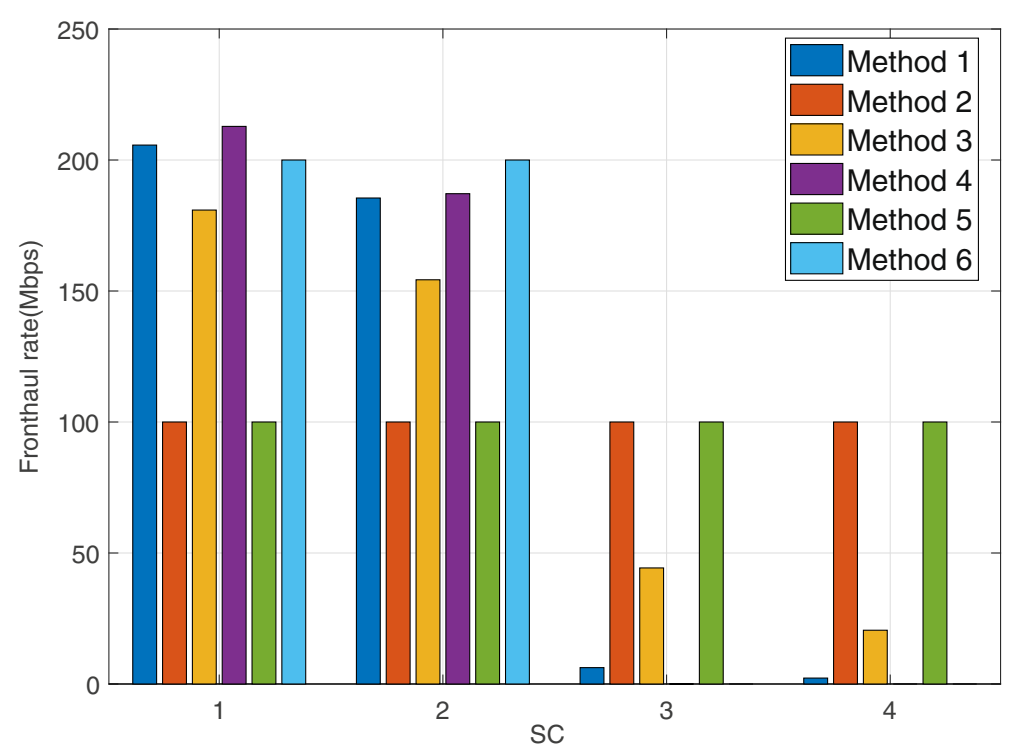

Fig. 3 Fronthaul rate allocation among four SCS

with method 4. For instance, the poorest channel condition SC 4 is almost shut down while the best channel SC 1 receive the most resources.

Conclusion 3: All methods, including Algorithm 1 in method 1 and Algorithm 2 in method 6, tend to allocate all fronthaul link capacity over all SCs. For example, the sum of the fronthaul rate of each subcarrier is almost $400 \mathrm{Mhz}$ in all methods. That means the fronthaul capacity boundary $T^{\text {total }}$ can be reached.

\subsubsection{Energy efficiency gain causing by different methods under Gaussian quantization}

Different from the settings above, bandwidth $B$ is set to $100 \mathrm{Mhz}$ and $N=32$ in this subsection. In an I.I.D Rayleigh fading channel model of six paths, the pass loss model is set as $L=30.6+36.7 \log _{10}(d)$. The distance between user and RRH is $50 \mathrm{~m}$. Other parameters are consistent with the settings in Subsection 3.3.1. In Fig. 4, parameter $P^{\text {total }}$ remains invariant when $T^{\text {total }}$ keeps unchanged in Fig. 5.

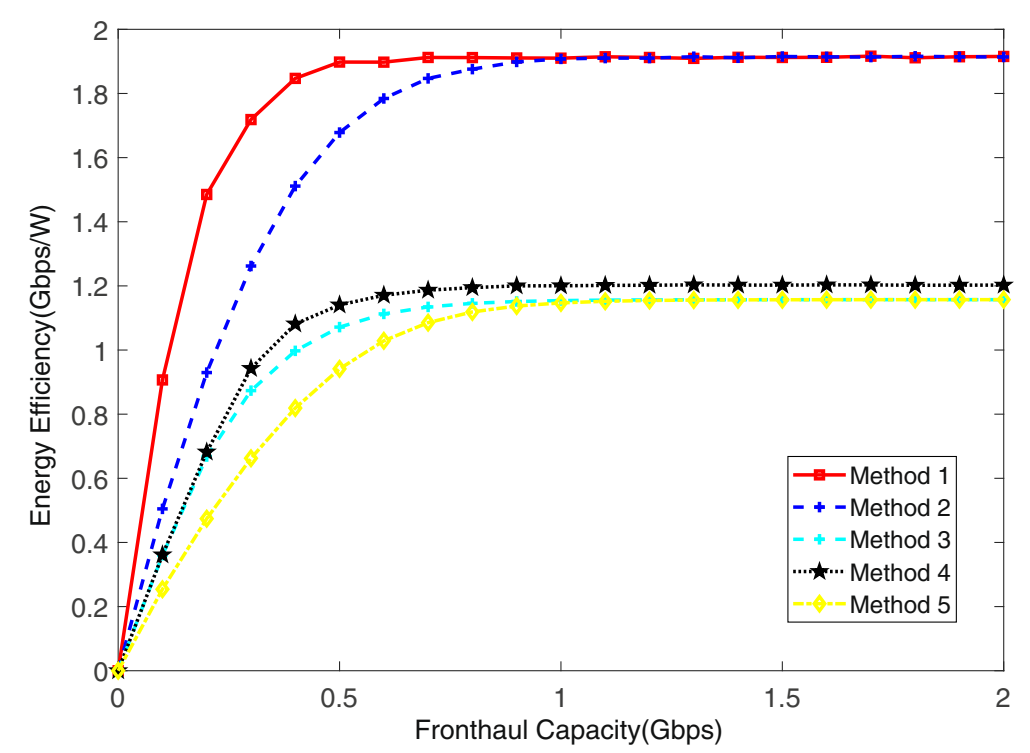

Fig. 4 Performance of various schemes under GQ versus fronthaul capacity 


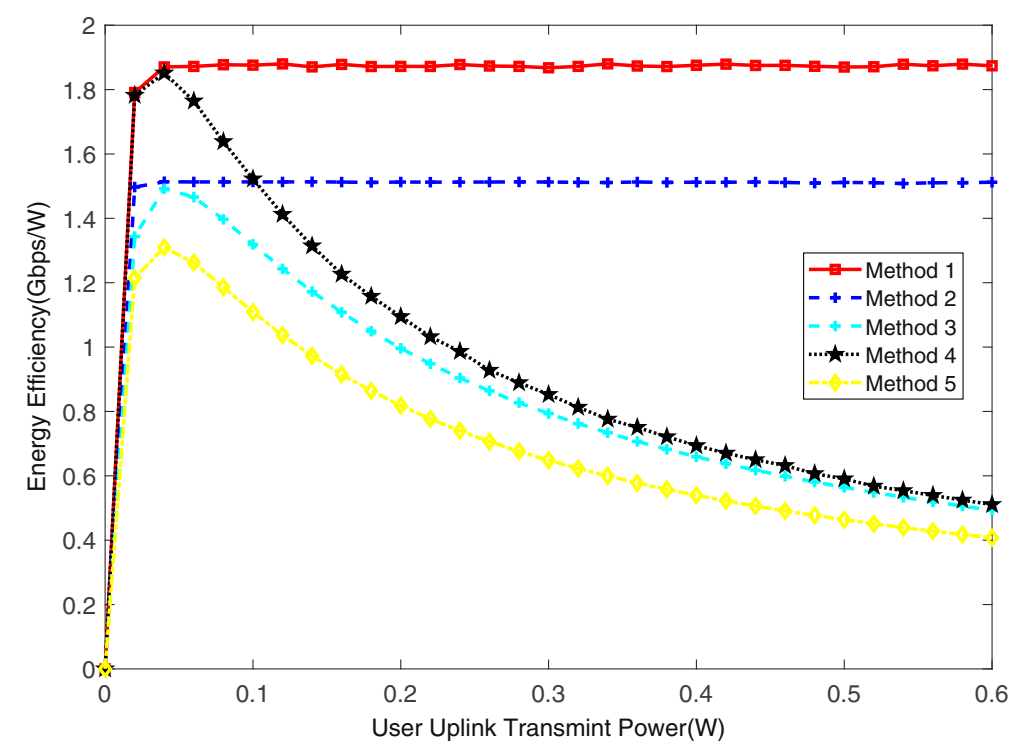

Fig. 5 Performance of various schemes under GQ versus power

In Figs. 4 and 5, some useful conclusions could be obtained as follows:

Conclusion 1: Fig. 4 shows Algorithm 1 proposed in this paper always achieves better EE performance compared with other methods. When $T^{\text {total }}<1 \mathrm{Gbps}$, the performance gain of Algorithm 1 is more obvious. However, when $T^{\text {total }}$ is continuously growing, the performance gain from power optimization only approximates Algorithm 1. The reason is that each symbol can be quantized by enough bits when $T^{\text {total }}$ is sufficiently large. Therefore, the effect of specific fronthaul allocation method is smaller. It shows Algorithm 1 works better for limited fronthaul capacity Cloud-RAN scenes. Because the channel conditions of RRHs and users are different, the performance of the scheme for average allocating resources is almost the worst.

Conclusion 2: Fig. 4 also indicates method 2 always produces more gain than method 3 . That is because optimizing users' power has more impacts on objective function than optimizing fronthaul capacity. Figure 5 shows that the proposed Algorithm 1 could realize better EE performance, allocate power on each SC effectively, and optimize two variables $p_{n}$ and $T_{n}^{(G)}$ jointly, when $P^{\text {total }}$ is growing. In Fig. 5, the maximum EE gradually grows to a maximum value and stops growth with $P^{\text {total }}$ increases when $T^{\text {total }}$ keeps unchanged.

\subsubsection{The effect of different parameter $\tau$ on EE gain for USQ method}

In this part, the simulation parameters are the same as the settings in part 3.3.2. Compared with problem (P1), problem (P2) for USQ method has an integer restriction, which is $T_{n}^{(U)}=2 B D_{n}^{(U)} / N, D_{n} \in\{1,2, \ldots\}, \forall n \in \mathcal{N}$. Figure 6 assumes stationary $P^{\text {total }}$ when $T^{\text {total }}$ is fixed in Fig. 7. In this subsection, the effect of different parameter $\tau$ is analyzed.

From Figs. 6 and 7, the following conclusions can be obtained:

Conclusion 1: When $\tau=0$, that means the restriction $T_{n}^{(U)}=2 B D_{n}^{(U)} / N, D_{n} \in\{1,2, \ldots\}$ does not work and the EE obtained could reach the upper bound. Meanwhile, $\tau=1$ indicates all $N T_{n}^{(U)} / 2 B$ is set to a large integer as possible. Because objective function of (P2) is monotonically increasing over $T_{n}^{(U)}, \tau=1$ causes the lower bound of EE.

Conclusion 2: The performance of the EE obtained by the USQ method is lower than that obtained by GQ method, under the same simulation parameters. This gap is understandable because the GQ method is theoretically optimal and often treated as a performance upper bound. However, in the actual scenario, the GQ method is difficult to achieve. When $T^{\text {total }}$ is large enough, the EE-USQ approaches the theoretical EE-GQ bound.

\section{New algorithms for multi-RRH scenario}

During this subsection, authors extend the special singleRRH scenario to general multi-RRH scenario, when $K>1$ and $M>1$. We assume $\Omega_{k}$ as the allocated SCs to single user $k$. Two corresponding restrictions are $k$ th user's maximum UL transmit power $P_{k}^{\text {total }}$ and $m$ th RRH's maximum 


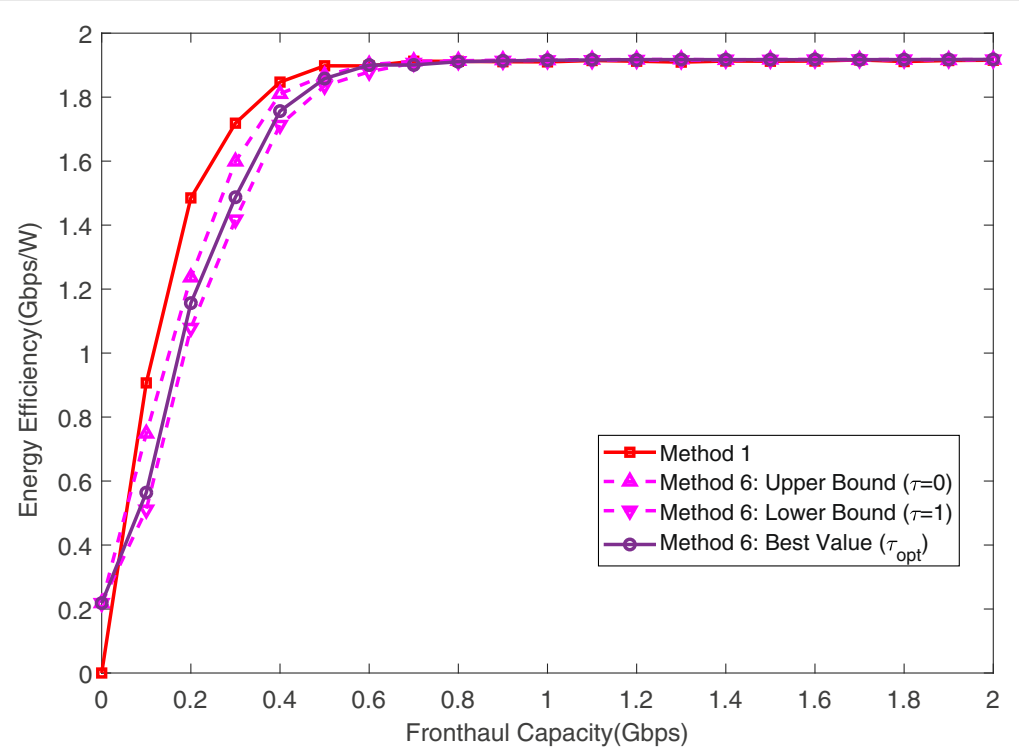

Fig. 6 The effect of $\tau$ (tau) under fixed $P^{\text {total }}$

fronthaul capacity $T_{m}^{\text {total }}$. Based on two different quantization schemes, the optimization models in multi-RRH scenario are as follows.

$$
\text { (P3) : } \max _{\left\{p_{k, n}, T_{m, n}^{(G)}\right\}} \frac{\frac{B}{N} \sum_{k=1}^{K} \sum_{n \in \Omega_{k}} \log _{2}\left(1+\sum_{m=1}^{M} \Lambda_{m}\right)}{\sum_{k=1}^{K} \sum_{n \in \Omega_{k}} p_{k, n}+P_{c}}
$$

$$
\begin{aligned}
& \sum_{n=1}^{N} T_{m, n}^{(G)} \leq T_{m}^{\text {total }}, \forall m \in \mathcal{M} \\
& \sum_{n \in \Omega_{k}} p_{k, n} \leq P_{k}^{\text {total }}, \forall k \in \mathcal{K}
\end{aligned}
$$

where

$$
\Lambda_{m}=\left(\left|h_{m, k, n}\right|^{2} p_{k, n}\right) /\left(\sigma_{m, n}^{2}+\frac{\left|h_{m, k, n}\right|^{2} p_{k, n}+\sigma_{m, n}^{2}}{2^{N T_{m, n}^{(G)} / B}-1}\right)
$$

s.t.

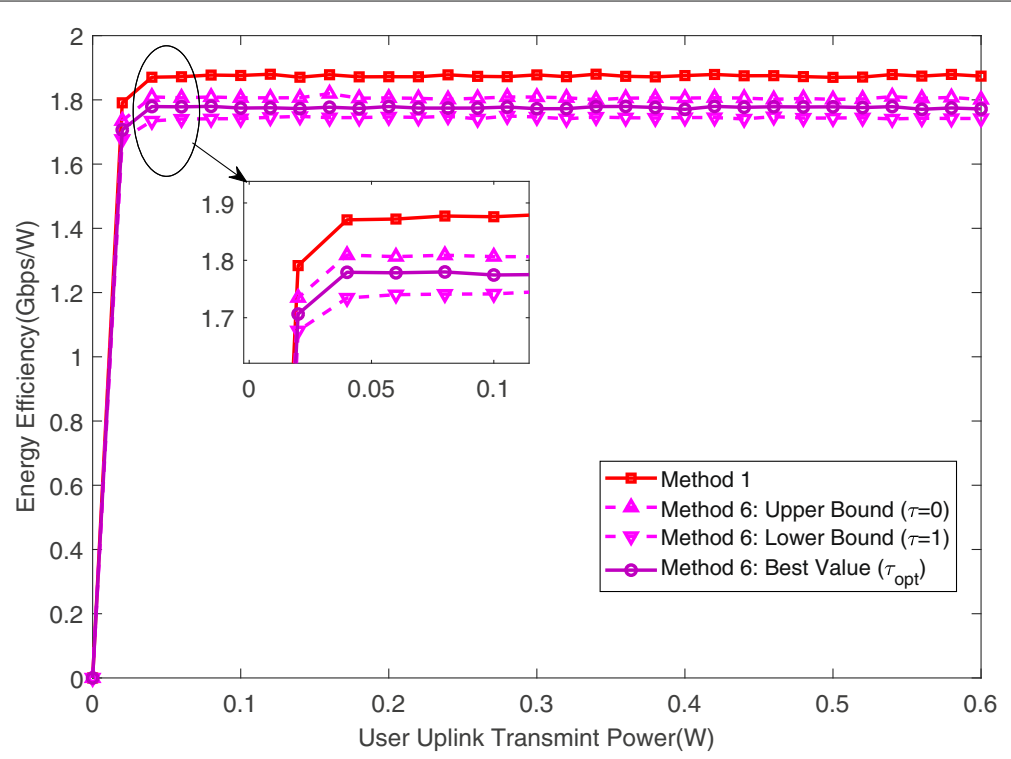

Fig. 7 The effect of $\tau$ (tau) under fixed $T^{\text {total }}$ 


$$
\text { (P4) : } \max _{\left\{p_{k, n}, T_{m, n}^{(U)}\right\}} \frac{\frac{B}{N} \sum_{k=1}^{K} \sum_{n \in \Omega_{k}} \log _{2}\left(1+\sum_{m=1}^{M} \Theta_{m}\right)}{\sum_{k=1}^{K} \sum_{n \in \Omega_{k}} p_{k, n}+P_{c}}
$$

s.t.

$$
\begin{aligned}
& \sum_{n=1}^{N} T_{m, n}^{(U)} \leq T_{m}^{\mathrm{total}}, \forall m \in \mathcal{M} \\
& \sum_{n \in \Omega_{k}} p_{k, n} \leq P_{k}^{\mathrm{total}}, \forall k \in \mathcal{K} \\
& T_{m, n}^{(U)}=2 B D_{m, n}^{(U)} / N, D_{m, n} \in\{1,2, \ldots\}, \forall m \in \mathcal{M}, \forall n \in \mathcal{N}
\end{aligned}
$$

where

$\Theta_{m}=\left|h_{m, k, n}\right|^{2} p_{k, n} /\left(\sigma_{m, n}^{2}+\frac{3\left(\left|h_{m, k, n}\right|^{2} p_{k, n}+\sigma_{m, n}^{2}\right)}{2^{N T_{m, n}^{(L)} / B}}\right)$

The similar optimization problems in general scenario for multi-user are more complex. And it is difficult to utilize fractional programming and Lagrangian dual decomposition directly. To address the non-convex and non-linear optimization problems, authors come up with a modified particle swarm optimization (M-PSO) algorithm. Actually, the M-PSO method also could be used for a singleuser scenario, but its effect is not good, because M-PSO is a heuristic algorithm, which is suitable for dealing with complex problems. For the single-user scenario which has been simplified, its performance is not significantly better than Algorithm 1. And M-PSO algorithm can only give the optimal strategy scheme within the allowable error range through simulation. However, the analytical expression of the optimal scheme cannot be given theoretically. Instead, we could give the analytical expression in Algorithm 1.

\subsection{The proposed M-PSO algorithm for P3}

Particle swarm optimization (PSO), originally got the inspiration from the movement feature of fish and bird, is a population-based approach to solve optimization problems by changing the candidate solution $[25,26]$. In PSO system, particles are moving around in the multidimensional search-space for particle's moving, according to the mathematical rule. Every particle adjusts its position based on its local best known position in the search history, but is also directed to the most known optimal positions of other particles. In the standard PSO algorithm, the velocity and position of single particle change are as follows:

$$
\begin{aligned}
& v_{q d}^{i+1}=w v_{q d}^{i}+c_{1} \xi\left(z_{q d}^{i}-x_{q d}^{i}\right)+c_{2} \rho\left(s_{g d}^{i}-x_{q d}^{i}\right) \\
& x_{q d}^{i+1}=x_{q d}^{i}+v_{q d}^{i+1}
\end{aligned}
$$

where

$Q_{\max }, 1 \leq q \leq Q_{\max }$ : The particle number;

$D, 1 \leq d \leq D$ : The space dimension;

$I_{\max }, 1 \leq i \leq I_{\max }$ : Maximum iterations;

$\mathbf{x}_{q}=\left(x_{q 1}, x_{q 2}, \ldots, x_{q D}\right)$ : Position information of particle $q$ in the D dimension;

$\mathbf{v}_{q}=\left(v_{q 1}, v_{q 2}, \ldots, v_{q D}\right)$ : Velocity information of particle $q$ in the D dimension;

$\mathbf{z}_{q}=\left(z_{q 1}, z_{q 2}, \ldots, z_{q D}\right)$ : Best historic position information of particle $q$ in the $\mathrm{D}$ dimension;

$\mathbf{s}_{g}=\left(s_{g 1}, s_{g 2}, \ldots, s_{g D}\right):$ Best historic position information of all particles in the $\mathrm{D}$ dimension;

$c_{1}, c_{2}$ : The learning factors that determine the size of step;

$w$ : The inertial weight which determine the influences of particle's previous velocity on next velocity;

$\xi, \rho$ : Randomly distributed numbers between $[0,1]$;

As stated in the formula above, standard PSO is more suitable for solving the non-constrained problems. Therefore, it is necessary to design an improved M-PSO for (P3-P4). Based on [27], this subsection considers the application of asynchronous time-varying learning factors $c_{1}, c_{2}$ and linear time-varying weights $w$. To guarantee the particles have the best performance in searching for the global optimal value in the initial stage and good convergence in the final stage, the dynamic range of weight $\left[w_{\min }, w_{\max }\right]$ is further considered. The corresponding expression between the dynamic weight $w$ and iteration number $i$ is as follows.

$$
w^{i}=w_{\max }-\left(w_{\max }-w_{\min }\right) / I_{\max } \times i
$$

For the same reason, learning factors $c 1$ and $c 2$ are also redesigned. The learning factors of the $i$ th iteration could be represented:

$$
\begin{aligned}
& c_{1}^{i}=\left(c_{1}^{\text {final }}-c_{1}^{\text {initial }}\right) \times i / I_{\text {max }}+c_{1}^{\text {initial }} \\
& c_{2}^{i}=\left(c_{2}^{\text {final }}-c_{2}^{\text {initial }}\right) \times i / I_{\text {max }}+c_{2}^{\text {initial }}
\end{aligned}
$$

where $c_{1}^{\text {initial }}, c_{1}^{\text {final }}, c_{2}^{\text {initial }}, c_{2}^{\text {final }}$ are the initial and final values of $\mathrm{C} 1$ and $\mathrm{C} 2$, respectively. In this paper, we select the following variable assignments $w_{\max }=1, w_{\min }=0.1$, $c_{1}^{\text {initial }}=c_{2}^{\text {final }}=4, c_{1}^{\text {final }}=c_{2}^{\text {initial }}=0.1$, for better convergence.

In Fig. 8, a numerical example is provided to prove the modified PSO algorithm has obvious advantages over standard PSO in seeking global optimal solution. The fronthaul capacity $T^{\text {total }}$ is fixed as 2 Gbps and UL transmission power $P^{\text {total }}$ is assumed as $0.2 \mathrm{~W}$. We set $M=8$, $K=8, P_{c}=0.5 \mathrm{~W}, B=150 \mathrm{Mhz}, N=32$. For convenience, each user is pre-assigned $N / K=4$ SCs. In an I.I.D 


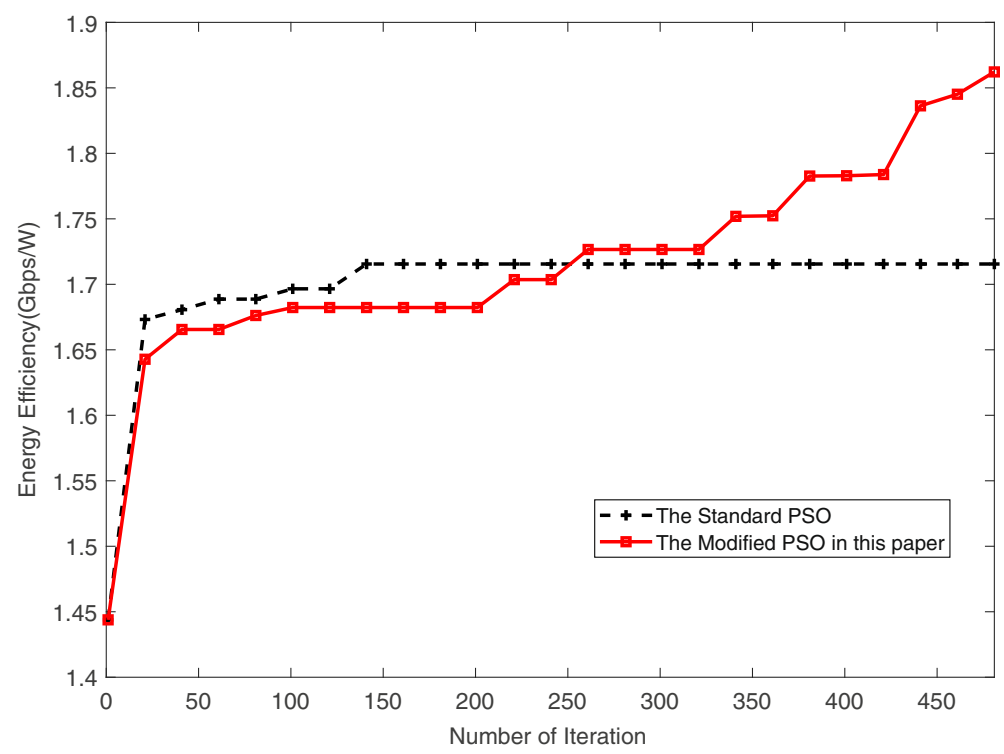

Fig. 8 The comparison between proposed M-PSO and standard PSO

Rayleigh fading channel model of six paths, we distribute RRHs and use randomly in a circle with a radius of $100 \mathrm{~m}$, when the pass loss model is set as $L=30.6+36.7 \log _{10}(d)$. Two different PSO algorithms have the same particle number $Q_{\max }=250$ and maximum iterations number $I_{\max }=1000$. Figure 8 reveals the proposed modified PSO algorithm can achieve better EE performance compared with standard PSO when $Q_{\max }, I_{\max }$ keep the same.

According to experimental tests in Appendix C, proper parameters $Q_{\max }=2000, I_{\max }=2000$ are chosen to offer good tradeoff between sufficiently good EE performance and sufficiently short searching time.

Therefore, a new algorithm of M-PSO is designed for (P3).

In step 2, $\mathbf{x}_{q}^{1}=\left(\left\{p_{k, n}\right\}_{q},\left\{T_{m, n}^{(G)}\right\}_{q}\right), \mathbf{v}_{q}^{1}=\left(\left\{v p_{k, n}\right\}_{q}\right.$, $\left.\left\{v T_{m, n}^{(G)}\right\}_{q}\right)$ are the initial position and velocity of $q$ th particle. The utility function of $\mathbf{F}$ is designed according to the objective function in (39) in step 3.

\subsection{The proposed M-PSO algorithm for P4}

To solve problem (P4), the utility function of $\mathbf{F}$ in Algorithm 3 should be set as the objective function in (43). In addition, particle position $\mathbf{x}_{q}^{i+1}$ should not only satisfy the constraint in $(44,45)$, but also ensure integer constraint in (46). Details of the specific adjustment of $\mathbf{x}_{q}^{i+1}$ in step 9 for USQ is similar to that in Algorithm 3.

\subsection{Multi-RRH scenario: numerical experiment}

Under two different quantization schemes, i.e., GQ and USQ, numerical examples are provided to evaluate the
Algorithm 3 Proposed M-PSO Algorithm for Jointly Optimization Under Gaussian Quantization for Problem (P3)

1: Input $w_{\min }, w_{\max }, c_{1}^{\text {initial }}, c_{2}^{\text {final }}, c_{1}^{\text {final }}$, etc;

2: Randomly initialize $\mathbf{x}_{q}^{1}=\left(\left\{p_{k, n}\right\}_{q},\left\{T_{m, n}^{(G)}\right\}_{q}\right), \mathbf{v}_{q}^{1}=$ $\left(\left\{v p_{k, n}\right\}_{q},\left\{v T_{m, n}^{(G)}\right\}_{q}\right)$ under constraint (40) (41);

3: Set $\mathbf{z}_{q}^{1} \leftarrow \arg \max \mathbf{F}\left(\mathbf{x}_{q}^{1}\right), \mathbf{s}_{g} \leftarrow \arg \max \mathbf{F}\left(\mathbf{z}_{q}^{1}\right)$ according to the objective function in (40) (41);

4: For $1 \leq i \leq I_{\max }$

5: For $1 \leq q \leq Q_{\max }$

6: $\quad \mathbf{x}_{q}^{i+1}=\mathbf{x}_{q}^{i}+\mathbf{v}_{q}^{i}$

7: $\quad$ IF $\mathbf{x}_{q}^{i+1}$ penetrate the borders in (40), (41) Reinstall $\mathbf{x}_{q}^{i+1}$;

When $\mathbf{x}_{1}^{i+1}$ can satisfies the constraints

9: When $\mathbf{x}_{1}^{i+1}$ can satisfies the constraints

10: $\quad$ Update $\mathbf{z}_{q}^{i+1} \leftarrow \arg \max _{\left\{\mathbf{x}_{q}^{i+1}\right\}} \mathbf{F}\left(\mathbf{x}_{q}^{i+1}\right)$, if

$\mathbf{F}\left(\mathbf{x}_{q}^{i+1}\right)>\mathbf{z}_{q}^{i}$

11: $\quad$ Update $\mathbf{s}_{g} \leftarrow \arg \max _{\left\{\mathbf{z}_{q}^{i+1}\right\}} \mathbf{F}\left(\mathbf{z}_{q}^{i+1}\right)>\mathbf{F}\left(\mathbf{z}_{q}^{i}\right)$

12: $\quad$ Update $\mathbf{v}_{q}^{i+1}=w^{i} \mathbf{v}_{q}^{i}+c_{1}^{i} \xi\left(\mathbf{z}_{q}^{i}-\mathbf{x}_{q}^{i}\right)+$ $c_{2}^{i} \rho\left(\mathbf{s}_{g}-\mathbf{x}_{q}^{i}\right)$ based on (48-52);

13: $\quad$ End When

14: $\quad i=i+1$;

15: $\quad$ End For

16: End For

17: Output $\mathbf{x}_{q}^{I_{\max }}, \mathbf{F}\left(\mathbf{x}_{q}^{I_{m} a x}\right)$; 
energy efficiency of different optimization methods. In Fig. 9, $P^{\text {total }}$ is fixed to $0.2 \mathrm{~W}$ when $T^{\text {total }}=0.8 \mathrm{Gbps}$ remains unchanged in Fig. 10. The rest of the relevant simulation parameters are set according to Fig. 8. We also compare energy efficiency under different resource allocation schemes in Figs. 9 and 10.

- Scheme 1 (Max EE Under GQ: Joint Opt By $M-P S O)$ : The proposed Algorithm 3 for joint optimization of user-centric energy efficiency under Gaussian quantization.

- Scheme 2 (Max EE Under GQ: Joint Opt By S-PSO in [25]): The user-centric EE optimized by standard PSO algorithm.

- Scheme 3 (Max EE Under GQ: Power Opt Only By $M-P S O)$ : Optimize power only in problem (P3) when fronthaul resources allocated equally.

- Scheme 4 (Max EE Under GQ: Fronthaul Opt Only By M-PSO): Optimize fronthaul capacity only in problem (P3), contrary to scheme 3.

- Scheme 5 (Max Throughput Under GQ: Interior-point Method in [8]): The algorithm presented in [8] for throughput maximization utilizing interior-point method.

- Scheme 6 (Max EE Under GQ: Equal Fronthaul Allocation and Power): Users allocate uplink transmit power over all SCs equally when RRHs allocate fronthaul link capacity over all SCs equally.

- Scheme 7 (Max EE Under USQ: Joint Opt M-PSO): In contrast to scheme 1 , the quantization method changed into uniform scalar quantization (USQ). During the period of signal processing, the received signal could be firstly converted to in-phase/quadrature (I/Q) parts. Secondly, every RRH uses positive integer bits to normalize I/Q parts. From an optimization point of view, the USQ method puts an integer limit condition on fronthaul rate $T_{m, n}$.

In Figs. 9 and 10, simulation results reveal the following conclusions:

Conclusion 1: All figures show the proposed M-PSO in Algorithm $37 \mathrm{y} 7$ realize better EE all the time, compared with S-PSO. Based on simulations of M-PSO algorithm in Appendix C, parameter $Q_{\max }=2000, I_{\max }=2000$ could offer good tradeoff between sufficiently good EE performance and sufficiently short searching time. In contrast, S-PSO could not implement a stable EE value, under the same parameters.

Conclusion 2: Fig. 9 indicates that the theoretical upper information EE obtained by GQ is superior to which obtained by USQ, when $T^{\text {total }}<2$ Gbps. Further, the EEUSQ nearly coincides with EE-GQ, when $T^{\text {total }}>2$ Gbps. This trend is consistent with the single-user scenario in Fig. 6. Besides, when $T^{\text {total }}$ is fixed in Fig. 10, there is a significant gap between the EE-GQ curve and the EEUSQ curve, which is similar to the conclusion of Fig. 7. At the same time, power optimization generate more gain than fronthaul optimization in Fig. 9. However, Fig. 10 gives the opposite case that fronthaul optimization is better than power optimization, meanwhile $T^{\text {total }}$ is fixed and $P^{\text {total }}<0.1 \mathrm{~W}$.

Conclusion 3: Both two figures shows that the improved M-PSO could realize better EE than which obtained by all other schemes. The similar conclusion is that

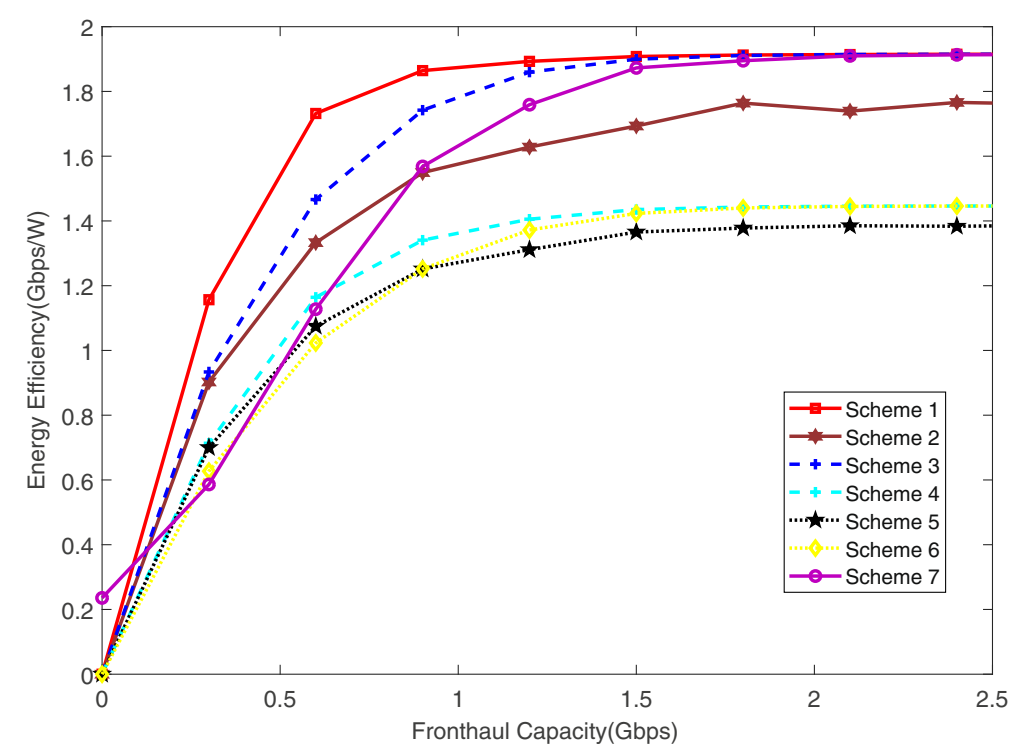

Fig. 9 Performance of various schemes versus fronthaul capacity for multi-RRH scenario 


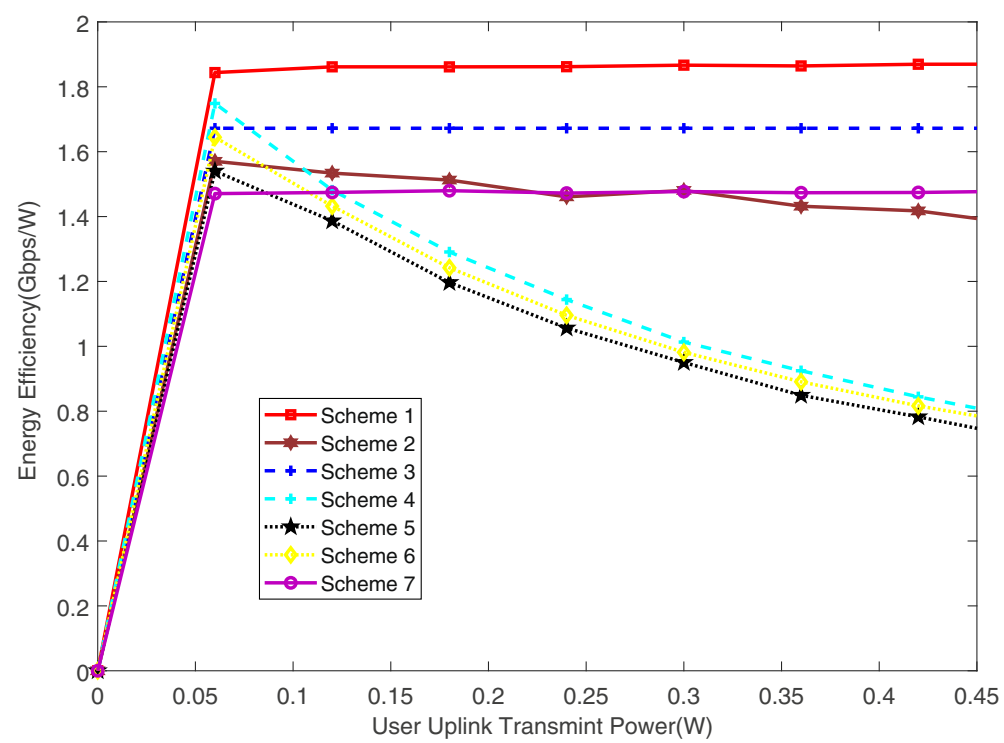

Fig. 10 Performance of various schemes versus power for multi-RRH scenario

performance gain achieved by Algorithm 3 is not as as significant as the power optimization only, when $T^{\text {total }}$ increases. Obviously, the corresponding $T^{\text {total }}$ of intersection between scheme 1 and scheme 3 is bigger than that obtained in a single scenario. That illustrates M-PSO algorithm is more suitable for a multi-user case, because fronthaul capacity constraints is more serious.

\section{Conclusion}

In this article, authors study the joint optimization problem to improve energy efficiency of users in Cloud-RAN system, under two quantization methods. The main corresponding restrictions are user's UL transmission power and RRH's fronthaul capacity. Compared with existing work, this paper firstly take into account "signal quantization" and "fronthaul capacity constraint" in user-centric EE maximization of OFDMA-based Cloud-RAN. For single-user scenario, the related algorithms are formulated to maximize the EE of users by optimizing power control and fronthaul capacity allocation. Then we extend single-RRH scenario to the more general scenario of multiple RRHs and resolve the more complex non-convex and non-linear problems by devising M-PSO algorithms. Simulation results demonstrate that the proposed schemes in this paper outperform conventional solutions and improve EE of users obviously.

\section{Appendix}

\section{A. Proof of proposition 3.1}

Proof We denote subscript optimal as opt. In problem (15), the EE performance of mobile users $\zeta$ is non-negative with the optimal value $\zeta_{\text {opt }}=C\left(p_{n}^{o p t}\right) / P\left(p_{n}^{o p t}\right)$. An equivalent function is defined $F(\zeta)=\max _{\left\{p_{n}\right\}} C\left(p_{n}^{o p t}\right)-$ $\zeta P\left(p_{n}^{o p t}\right)$. According to [24], the problem (17) is equivalent to new transformed EE optimization

$$
\max _{\left\{p_{n}\right\}} C\left(p_{n}^{o p t}\right)-\zeta P\left(p_{n}^{o p t}\right)
$$

$F(\zeta)$ is strictly monotonic decreasing when $\zeta$ increases with $F(\zeta) \geq 0$.

Based on the decreasing characteristic of $F(\zeta)$, Algorithm 4 is raised to settle the transformed issue by updating $\zeta$ in the following algorithm.

When the non-zero duality between the optimal value of $\max C\left(p_{n}\right)-\zeta^{(i)} P\left(p_{n}\right)$ and its dual problem is nearly $\left\{p_{n}\right\}$

zero, the problem of the $i$ th inner loop could be solved by Lagrange dual decomposition method.

The Lagrange function of the problem in the $i$ th inner loop is as follows

$$
\begin{aligned}
& L\left(\left\{p_{n}\right\}, \lambda\right)=\frac{B}{N} \sum_{n=1}^{N} \log _{2}\left(1+\frac{\left|h_{n}\right|^{2} p_{n}}{\sigma_{n}^{2}+\frac{\left.||_{n}\right|^{2} p_{n}+\sigma_{n}^{2}}{\left.2^{N T_{n, o p t}^{(G}}\right)}}\right) \\
& -\zeta^{(i)}\left(\sum_{n=1}^{N} p_{n}+P_{c}\right)-\lambda^{(i)}\left(\sum_{n=1}^{N} p_{n}-P^{\text {total }}\right)
\end{aligned}
$$

where $\lambda$ is the dual vector associated with the total transmission power limitation in (18). For single SC, the subproblem can be expressed as 


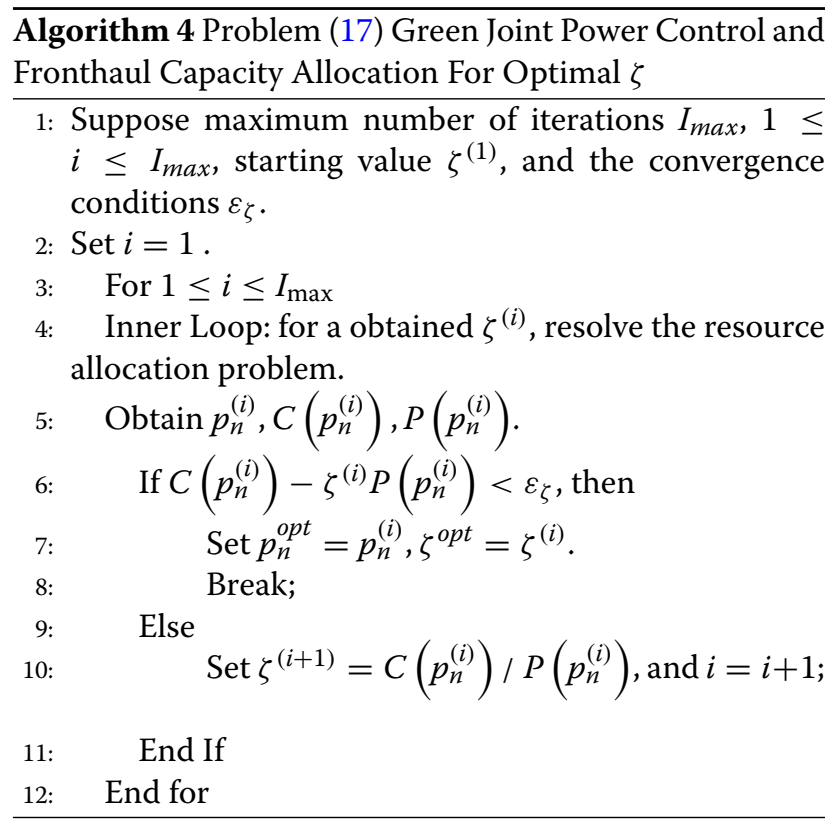

$$
\max _{p_{n} \geq 0} L_{n}\left(p_{n}\right)
$$

where

$L_{n}\left(p_{n}\right)=\frac{B}{N} \log _{2}\left(1+\frac{\left|h_{n}\right|^{2} p_{n}}{\sigma_{n}^{2}+\frac{\left|h_{n}\right|^{2} p_{n}+\sigma_{n}^{2}}{2^{N T_{n, o p t}^{(G)}-1}}}\right)-\left(\zeta^{(i)}+\lambda\right) p_{n}$

Since $L_{n}\left(p_{n}\right)$ is concave over $p_{n}$, its corresponding derivative over $p_{n}$ is shown below

$$
\begin{aligned}
& \frac{\partial L_{n}\left(p_{n}\right)}{\partial p_{n}}=\frac{B}{N \ln 2} \frac{\left|h_{n}\right|^{2} \sigma_{n}^{2}\left(2^{N T_{n, o p t}^{(G)} / B}-1\right)}{\left(2^{N T_{n, o p t}^{(G)} / B} \sigma_{n}^{2}+\left|h_{n}\right|^{2} p_{n}\right)\left(\sigma_{n}^{2}+\left|h_{n}\right|^{2} p_{n}\right)} \\
& -\left(\zeta^{(i)}+\lambda\right), \forall n
\end{aligned}
$$

By setting $\frac{\partial L_{n}\left(p_{n}\right)}{\partial p_{n}}=0$, then we have the following expression

$$
p_{n}^{2}+\frac{\left(2^{N T_{n, o p t}^{(G)} / B}+1\right) \sigma_{n}^{2}}{\left|h_{n}\right|^{2}} p_{n}+\frac{2^{N T_{n, o p t}^{(G)} / B} \sigma_{n}^{4}}{\left|h_{n}\right|^{4}}-\Pi=0, \forall n
$$

where

$$
\Pi=\frac{B}{\ln 2} \frac{\sigma_{n}^{2}\left(2^{N T_{n, o p t}^{(G)} / B}-1\right)}{N\left(\lambda^{(i)}+\zeta^{(i)}\right)\left|h_{n}\right|^{2}}
$$

By denoting $\alpha_{n}$ as (28), $\eta_{n}$ as (30), the above expression can be expressed as

$$
p_{n}^{2}+\alpha_{n} p_{n}+\eta_{n}=0, \forall n
$$

According to the relevant characteristics of a quadratic equation, to obtain the meaningful solution, we denote

$$
\alpha_{n}^{2}-4 \eta_{n} \geq 0
$$

and then we have the following expression

$$
\alpha_{n}^{2}-4 \eta_{n}=\frac{\sigma_{n}^{2}}{\left|h_{n}\right|^{2}}+4 \frac{1}{\ln 2 N\left(\lambda^{(i)}+\zeta^{(i)}\right)\left(2^{N T_{n, o p t}^{(G)} / B}-1\right)}>0
$$

So $\alpha_{n}^{2}-4 \eta_{n}$ always set up and the above quadratic equation always have the meaningful solution. At the same time, because $p_{n} \geq 0$, we set the constraint

$$
\eta_{n}<0
$$

By substituting $\eta_{n}$ in (30), we can further draw the following conclusion

$$
\frac{\left|h_{n}\right|^{2}}{\sigma_{n}^{2}}>\frac{\ln 2 N}{B} \frac{2^{N T_{n, o p t}^{(G)} / B}\left(\lambda^{(i)}+\zeta^{(i)}\right)}{2^{N T_{n, o p t}^{(G)} / B}-1}=f_{n}\left(T_{n, o p t}^{(G)}\right)
$$

Because the objective function in (17) is not increasing monotonically over $p_{n}$, the subgradient-based method is utilized to obtain in $\lambda$ [28]. The subgradient of the dual function is shown below

$$
\nabla \lambda^{(i+1)}=P^{\text {total }}-\sum_{n=1}^{N} p_{n}^{(i)}
$$

where $p_{n}^{(i)}$ means the allocated power at $\mathrm{SC} n$ of $i$ th iteration, $\nabla \lambda^{(i+1)}$ is the subgradient applied in $(i+1)$ th iteration. Therefore, the iterative formulas of dual variables in $(i+1)$ th iteration are shown below

$$
\lambda^{(i+1)}=\left[\lambda^{(i)}-\chi_{\lambda}^{(i+1)} \times \nabla \lambda^{(i+1)}\right]^{+}
$$

where $\chi_{\lambda}^{(i+1)}$ is the positive step size.

Proposition 3.1 is thus proved.

\section{B. Proof of Proposition 3.2}

Proof The Lagrangian of problem (27) can be expressed as

$$
\begin{aligned}
& L\left(\left\{T_{n}^{(G)}\right\}, \beta\right)=\frac{B}{N} \frac{\sum_{n=1}^{N} \log _{2}\left(1+\frac{\left|h_{n}\right|^{2} p_{n}^{o p t}}{\sigma_{n}^{2}+\frac{\left|h_{n}\right|^{2} p_{n}^{o p t}+\sigma_{n}^{2}}{2^{N T_{n}^{(G)} / B}-1}}\right)}{\sum_{n=1}^{N} p_{n}^{o p t}+P_{c}} \\
& -\beta\left(\sum_{n=1}^{N} T_{n}^{(G)}-T^{\text {total }}\right)
\end{aligned}
$$

where $\beta$ is the dual vector corresponding to fronthaul capacity constraint in problem (27). Similar to proof of proposition 3.1, for single SC, the associated subproblem 
is shown below

$$
\max _{T_{n}^{(G)} \geq 0} L_{n}\left(T_{n}^{(G)}\right), \forall n
$$

where

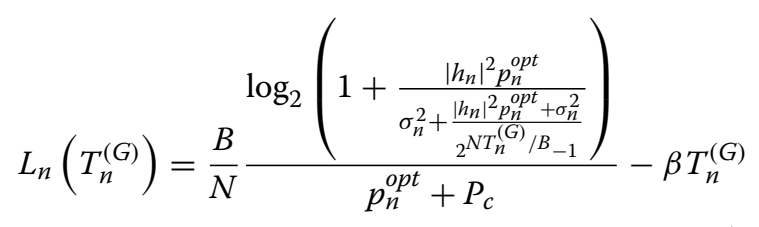

Since $L_{n}\left(T_{n}^{(G)}\right)$ is concave over $T_{n}^{(G)}$, its corresponding derivative over $T_{n}^{(G)}$ can be expressed as

$$
\frac{\partial L_{n}\left(T_{n}^{(G)}\right)}{\partial T_{n}^{(G)}}=\frac{1}{p_{n}^{\text {opt }}+P_{c}}\left(1-\frac{\sigma_{n}^{2} 2^{N T_{n}^{(G)} / B}}{\left|h_{n}\right|^{2} p_{n}^{o p t}+\sigma_{n}^{2} 2^{N T_{n}^{(G)} / B}}\right)-\beta
$$

By setting $\partial L_{n}\left(T_{n}^{(G)}\right) / \partial T_{n}^{(G)}=0$, then we have the following expression

$$
2^{N T_{n}^{(G)} / B}=\frac{\left|h_{n}\right|^{2} p_{n}^{o p t}}{\beta \sigma_{n}^{2}\left(p_{n}^{o p t}+P_{c}\right)}-\frac{\left|h_{n}\right|^{2} p_{n}^{o p t}}{\sigma_{n}^{2}}
$$

Since $T_{n}^{(G)} \geq 0$ always exists, to obtain the meaningful solution, we have

$$
\frac{\left|h_{n}\right|^{2} p_{n}^{o p t}}{\beta \sigma_{n}^{2}\left(p_{n}^{o p t}+P_{c}\right)}-\frac{\left|h_{n}\right|^{2} p_{n}^{o p t}}{\sigma_{n}^{2}}>1
$$

From the above expression of $T_{n}^{(G)}$, we can further derive

$T_{n}^{(G)}=\frac{B}{N} \log _{2}\left(\frac{\left|h_{n}\right|^{2} p_{n}^{o p t}}{\sigma_{n}^{2}}\right)+\frac{B}{N} \log _{2}\left(\frac{1}{\beta\left(p_{n}^{o p t}+P_{c}\right)}-1\right)$

$$
\beta<\frac{1}{p_{n}^{o p t}+P_{c}}
$$

Assume $v_{n}=\left|h_{n}\right|^{2} p_{n}^{\text {opt }} / \sigma_{n}^{2}$, we can obtain the conclusion in expression (25).

Under the constraint $\beta<\frac{1}{p_{n}^{o p t}+P_{c}}$ given above, optimal $\beta$ can be obtained when the fronthaul link capacity is tight in problem (27). The optimal $\beta_{\text {opt }}$ could be obtained by a bisection algorithm, as a solution to problem (27).

Proposition 3.2 is thus proved.
Table 1 Comparison between different $Q_{\max }$ and $I_{\max }$ in modified PSO algorithm

\begin{tabular}{llll}
\hline$Q_{\max }$ & $I_{\max }$ & Stable value of EE (Gbps/W) & Calculating time (h:m:s) \\
\hline 500 & 500 & 1.8669 & $0: 2: 09$ \\
750 & 500 & 1.8873 & $0: 3: 10$ \\
1500 & 500 & 1.890 & $0: 5: 41$ \\
2000 & 2000 & 1.9143 & $0: 45: 23$ \\
3000 & 3000 & 1.9145 & $2: 25: 01$ \\
4000 & 4000 & 1.9147 & $6: 06: 03$ \\
5000 & 5000 & 1.9147 & $14: 2: 5$ \\
\hline
\end{tabular}

\section{Comparison between different $Q_{\max }$ and $I_{\max }$ in modified PSO algorithm}

In Table 1, the simulation parameters is the same as which in Fig. 8. From this table, we can learn when we set $Q_{\max }=2000, I_{\max }=2000$ is suitable. Because when the number of particles $Q_{\max }$ and maximum number of iterations $I_{\max }$ continue to increase, the final EE stable value keeps the same while the total time calculating by MATLAB grows rapidly.

\section{Abbreviations}

Cloud-RAN: Cloud radio access network; BBU: Baseband unit; DL: Downlink; EE: Energy efficiency; Gbps: Gigabit byte per second; GQ: Gaussian quantization; M-PSO: Modified particle swarm optimization; OFDMA Orthogonal frequency division multiple access; PSO: Particle swarm optimization; $\mathrm{RRH}$ : Remote radio head; SC: Subcarrier; SNR: Signal to noise ratio; UE: User; UL: Uplink; USQ: Uniform scalar quantization

\section{Funding}

This work was supported by the 863 Program of China under Grant 2015-AA01A703, the National Natural Science Foundation of China under Grants 61422105, 61531011, and 61671144, the Research Project of Jiangsu Province under Grant BE2015156 and the Natural Science Foundation of Jiangsu Province for Colleges and Universities under Grants 17KJB510016.

\section{Authors' contributions}

YS completes the main work and updates the manuscript of this paper, as the first author. LY is the correspondence author of this paper. All authors read and approved the final manuscript.

\section{Competing interests}

The authors declare that they have no competing interests.

\section{Author details}

${ }^{1}$ School of Information Science and Engineering, Southeast University, 210096 Nanjing, China. ${ }^{2}$ School of Physical and Electronic Engineering, Jiangsu Normal University, 221116 Xuzhou, China.

\section{Publisher's Note}

Springer Nature remains neutral with regard to jurisdictional claims in published maps and institutional affiliations.

Received: 24 May 2018 Accepted: 17 July 2018

Published online: 02 August 2018

\section{References}

1. JG Andrews, S Buzzi, W Choi, et al, What Will 5G Be? IEEE J. Sel. Areas Commun. 32(6), 1065-1082 (2014)

2. M China, C-RAN: the road towards green RAN. White Paper. 2, 1-10 (2011)

3. A Checko, HL Christiansen, Y Ying, et al, Cloud RAN for Mobile Networks-A Technology Overview. IEEE Communications Surveys And Tutorials. IEEE Commun. Surv. Tutorials. 17(1), 405-426 (2015) 
4. R Wang, HL Hu, XM Yang, Potentials and Challenges of C-RAN Supporting Multi-RATs Toward 5G Mobile Networks. IEEE Access. 2, 1187-1195 (2014)

5. JH Tang, PT Wee, TQS Quek, Cross-Layer Resource Allocation With Elastic Service Scaling in Cloud Radio Access Network. IEEE Trans. Wirel. Commun. 14(9), 5068-5081 (2015)

6. SX Luo, R Zhang, TJ Lim, Downlink and Uplink Energy Minimization Through User Association and Beamforming in C-RAN. IEEE Trans. Wirel. Commun. 14(1), 494-508 (2015)

7. T Zhou, Z Liu, Qin D, et al, User Association With Maximizing Weighted Sum Energy Efficiency for Massive MIMO-Enabled Heterogeneous Cellular Networks. IEEE Commun. Lett. 21(10), 2250-2253 (2017)

8. L Liu, SZ Bi, R Zhang, Joint Power Control and Fronthaul Rate Allocation for Throughput Maximization in OFDMA-Based Cloud Radio Access Network. IEEE Trans. Commun. 63(11), 4097-4110 (2015)

9. CG Li, YS Li, Energy efficient design for multiuser downlink energy and uplink information transfer in 5G. Sci. China Inf. Sci. 59(2) (2016)

10. SH Park, O Simeone, O Sahin, S Shamai, Joint Decompression and Decoding for Cloud Radio Access Networks. IEEE Signal Process. Lett. 20(5), 503-506 (2013)

11. CG Li, K Song, DM Wang, Optimal remote radio head selection for cloud radio access networks. SCIENCE CHINA Information Sciences. 59(10) (2016)

12. Q Hou, SW He, YM Huang, et al, Energy efficient precoding design for cloud radio access networks. IET Commun. 11(12), 1864-1870 (2017)

13. BB Dai, W Yu, Energy Efficiency of Downlink Transmission Strategies for Cloud Radio Access Networks. IEEE J. Sel. Areas Commun. 30(4), 1037-1050 (2016)

14. KZ Wang, K Yang, CS Magurawalage, Joint Energy Minimization and Resource Allocation in C-RAN with Mobile Cloud. IEEE Trans. Mobile Comput. Early Access (2017)

15. L Li, Xu Chq, MX Tao, Resource Allocation in Open Access OFDMA Femtocell Networks. IEEE Commun. Lett. 1(6), 625-628 (2012)

16. YB Lin, TH Chiu, YT Su, Optimal and near-optimal resource allocation algorithms for OFDMA networks. IEEE Trans. Wirel. Commun. 8(8), 4066-4077 (2009)

17. Z Fei, CW Xing, N Li, et al, Leakage-based distributed minimum-mean-square error beamforming for relay-assisted cloud radio access networks. IET Commun. 8(11), 1883-1891 (2014)

18. N Li, ZS Fei, CW Xing, et al, Robust Low-Complexity MMSE Precoding Algorithm for Cloud Radio Access Networks. IEEE Commun. Lett. 18(5), 773-776 (2014)

19. YH Zhou, W Yu, Optimized Backhaul Compression for Uplink Cloud Radio Access Network. IEEE J. Sel. Areas Commun. 32(6), 1295-1307 (2014)

20. Y Linde, A Buzo, An Algorithm for Vector Quantizer Design. IEEE Trans. Commun. 28(1), 84-95 (1980)

21. A Goldsmith, Wireless communications. (Cambridge University Press, 2005)

22. R Zhao, YM Huang, Wang W, et al, Ergodic Achievable Secrecy Rate of Multiple-Antenna Relay Systems with Cooperative Jamming. IEEE Trans. Wirel. Commun. 15(4), 2537-2551 (2016)

23. El Gamal, YH Kim, Network information theory. (Cambridge University Press, 2011)

24. W Dinkelbach, On nonlinear fractional programming. Manag. Sci. 13(7) (1967)

25. J Kennedy, Particle swarm optimization. Encyclopedia of machine learning. (Springer, Boston, 2011), pp. 760-766

26. R Eberhart, J Kennedy, in Proceedings of the Sixth International Symposium on MHS, Nagoya. A new optimizer using particle swarm theory (IEEE, 1995), pp. 39-43

27. A Ratnaweera, Self-organizing hierarchical particle swarm optimizer with time-varying acceleration coefficients. IEEE Trans. Evol. Comput. 8(3), 240-255 (2004)

28. S Boyd, L Vandenberghe, Convex optimization. (Cambridge University Press, 2004)

\section{Submit your manuscript to a SpringerOpen ${ }^{\circ}$ journal and benefit from:}

- Convenient online submission

Rigorous peer review

- Open access: articles freely available online

- High visibility within the field

- Retaining the copyright to your article

Submit your next manuscript at $>$ springeropen.com 